\title{
A systematic review and meta-analysis of acupuncture for improving learning and memory ability in animals
}

\author{
Kai-Yu Huang ${ }^{1 \dagger}$, Shuang Liang ${ }^{1 \dagger}$, Mei-Ling Yu' ${ }^{1}$, Shu-Ping Fu ${ }^{1,2}$, Xia Chen ${ }^{1}$ and Sheng-Feng $\mathrm{Lu}^{1,2^{*}}$
}

\begin{abstract}
Background: Memory loss is the most prominent symptoms of brain aging, but there is currently no evidencebased treatment strategy. Acupuncture has been widely used in China and the effectiveness for improving learning and memory has been mentioned in previous studies. We conducted this systematic review and meta-analysis to evaluate the effectiveness of acupuncture for improving learning and memory in animal experiments.

Methods: We searched Pubmed, Embase, Ovid Medline(R), the China National Knowledge Infrastructure (CNKI), Chinese Science and Technology Periodical Database (VIP) and Wanfang data Information Site to collect studies published up to December 2015. Study quality for each included article was evaluated according to the CAMARADES 10-item checklist. Outcome measure is Morris water maze. A meta-analysis was conducted according to the Cochrane systematic review method by using RevMan 5.3 software.
\end{abstract}

Results: Forty-two studies involving 944 animals were included. The quality score of the studies ranged from 2 to 8 , with a mean of 5.3. Meta-analysis results showed that 24 studies reported significant effect of acupuncture for decreasing escape latency $(-3.00,95 \% \mathrm{Cl}:-3.78 \sim-2.23, P<0.00001)$, 14 studies reported significant effect of acupuncture for increasing frequency of cross platform (2.57, $95 \% \mathrm{Cl}: 1.92 \sim 3.22, P<0.00001)$, and 7 studies reported significant effect of acupuncture for increasing time in target quadrant (2.00, $95 \%$ Cl: $1.10 \sim 2.91$, $P<0.00001)$ compared with the control group.

Conclusions: These findings show acupuncture has a potential role in improving learning and memory ability in animal models, suggesting it as a candidate therapy for memory loss of aged brain.

Keywords: Acupuncture, Learning, Memory, Meta-analysis

Abbreviations: 4-VO, 4-vessel occlusion; 6-OHDA, 6-OH-dopamine; AD, Alzheimer's disease; CFS, Chronic fatigue syndrome; $\mathrm{Cl}$, Confidence interval; CMS, Chronic mild stimulation; CNKI, China National Knowledge Infrastructure; MCAO, Middle cerebral artery occlusion; MDA, Malondialdehyde; MMP, Matrix metalloproteinase; OVX, Ovariectomy; PD, Parkinson's disease; PTSD, Post-traumatic stress disorder; SD, Sprague-Dawley; SMD, Standard mean difference; SOD, Superoxide dismutase; SPS, Single prolonged stress; STZ, Streptozotocin; VD, Vascular dementia; VIP, Chinese Science and Technology Periodical Database; VPA, Sodium valproate; WD, Wilson disease

\footnotetext{
* Correspondence: lushengfeng@njucm.edu.cn

${ }^{\dagger}$ Equal contributors

${ }^{1}$ The No.2 Clinical Medical College, Nanjing University of Chinese Medicine, Nanjing 210023, China

${ }^{2}$ Key Laboratory of Acupuncture and Medicine Research of Ministry of

Education, Nanjing University of Chinese Medicine, Nanjing 210023, China
} International License (http://creativecommons.org/licenses/by/4.0/), which permits unrestricted use, distribution, and reproduction in any medium, provided you give appropriate credit to the original author(s) and the source, provide a link to the Creative Commons license, and indicate if changes were made. The Creative Commons Public Domain Dedication waiver (http://creativecommons.org/publicdomain/zero/1.0/) applies to the data made available in this article, unless otherwise stated. 


\section{Background}

Learning and memory are the most basal and important higher nervous functions and closely related to each other [1]. Learning means accepting information from the outside environment and memory refers being able to use this information at a later date [2-4]. As a symptom, learning and memory impairment often appears together in some diseases, such as Alzheimer's disease, vascular dementia, diabetes, autism, and so on [5-8]. With the increasing of living pressure and changing in life style, learning and memory impairment as the important embodiment of brain dysfunction have become one of the most important factors that affect people's lives $[1,2]$. The treatment based on western medical science for most learning and memory impairment only relieves the symptoms and delays the progression of disease $[9,10]$. Moreover, it also has some side effects caused by long time treatment [11].

As a kind of economical and side-effect free natural remedies, acupuncture has been used in China widely for over 2000 years [12]. The effect of acupuncture on encephalopathy has been recognized internationally. More and more studies have been published to confirm the effectiveness of acupuncture for improving learning and memory [13-16]. However, to some extent, the small sample size makes it hard to draw firm conclusions.

Up to now, there have been no systematic reviews to analyze the effectiveness of acupuncture for improving learning and memory. Reviews based on animal data could make trails' planning more perfect, increase the odds of success of future clinical trials and assist to decide what is valuable in further research [17]. Additionally, animal experiment can make us better understand the mechanism of acupuncture on learning and memory and guide the future clinical study. Therefore, we conducted a systematic review and meta-analysis of the effectiveness of acupuncture for improving learning and memory in animal experiments to provide suggestions for future animal experiments and clinical trials.

\section{Methods}

\section{Search strategy}

The following electronic databases were searched: Pubmed, Embase, Ovid Medline(R), China National Knowledge Infrastructure (CNKI), Chinese Science and Technology Periodical Database (VIP) and Wanfang data Information Site. The publication time is from the inception of each database up to December 2015. The languages were limited to English and Chinese. Search terms consisted of two groups: intervention (acupuncture and other related terms) and object (learning and memory and other related terms). All searches were limited to animals. We combined the results of all searches and then removed the duplicates. We also tried to get additional records identified through other sources.

\section{Inclusion criteria}

They were included if the following criteria were met:

(1)Subjects: Animal models of learning and memory impairment were included.

(2)Interventions: Acupuncture was the main therapy and only included manual acupuncture and electroacupuncture.

(3).Outcomes: Morris water maze test was the primary outcome to explore the effectiveness of acupuncture groups and the difference between control groups and acupuncture groups. The Morris maze test is arguably the preferred test for assessing learning and memory in basic research. As a classic test, it has been accepted and used widely in most related animals experiments.

(4) Language: Chinese and English articles.

\section{Exclusion criteria}

They were excluded if the following criteria were met: (1) Scalp acupuncture, auricular acupuncture, moxibustion and other forms of acupuncture; (2) studies that included Chinese herbal medicine or Western medicine; (3) studies that compared different acupuncture techniques or different acupoints; (4) studies without control group; (5) duplicate publications.

\section{Study selection and data extraction}

According to the above design, one reviewer $(\mathrm{KYH})$ searched those databases and listed the titles of all articles. Two evaluators (KYH and SL) assessed the eligibility of these articles and made decision on every research (inclusion or exclusion) independently. If they did not reach the same decision, the concerned articles were discussed with a third reviewer (SFL).

Two reviewers (KYH and SL) extracted data independently from each study. The database included: (1) basic information, including publication year, the first author's name and model of learning and memory impairment; (2) individual data, including the number of animals, species and weight in acupuncture group and control group; (3) information on treatment, including timing and duration for treatment and method of treatment procedure; (4) the results of Morris water maze test. If outcomes were presented at different time points, we extracted data from the last time point. Differences of extracted data were solved after discussion with a third reviewer (SFL). 


\section{Quality assessment}

We evaluated the methodological quality of the included studies by a ten-item scale [12]: (1) publication in a peer-reviewed journal; (2) statements describing control of temperature; (3) random allocation to treatment or control; (4) blinded building of model; (5) assessment whether building model is successful; (6) blinded assessment of outcome;(7) use of anesthetic without significant intrinsic neuroprotective activity; (8) sample size calculation; (9) compliance with animal welfare regulations; (10) declared any potential conflict of interest. Each item of the ten-item scale was attributed to one point. Based on this, each study had a quality score from zero to ten. The higher the score is, the better the article's quality is.

Two reviewers (KYH and SL) extracted data independently and assessed study quality. Disagreements were solved after discussion with a third reviewer (SFL).

\section{Statistical analysis}

Some results of Morris water maze test including escape latency, frequency of cross platform and time in target quadrant were considered as continuous data. Standard mean difference (SMD) was given, which was an estimate of the combined effect sizes. Publication bias was assessed with a funnel plot. Moreover, to explore the impact of factors affecting the outcome measures, we analyzed the specific subgroups based on escape latency and frequency of cross platform for the following factors: manual acupuncture and electroacupuncture, articles published or unpublished, species of animals, different ways to make Alzheimer's disease (AD) model and different ways to make vascular dementia (VD) model.

The meta-analysis was performed with RevMan 5.3 software. The confidence interval (CI) was established at $95 \%$, and $\mathrm{P}$ values of less than 0.05 were considered statistically significant. For the assessment of heterogeneity, the $\mathrm{I}^{2}$ statistic and $\chi^{2}$ distribution were used.

\section{Results}

\section{Study inclusion}

Initially, 1421 records were searched from six databases. After removing duplicates, the records were decreased to 875 . Based on titles and abstracts of records, we excluded 584 papers with reasons, such as not an animal experiment, case report or review, not related to learning and memory, and so on. The 291 remaining articles were downloaded for further selection. Due to republications, not using the Morris water maze test, comparing with other forms of acupuncture or Chinese herbs, and so on, 250 articles were excluded. Eventually, 42 studies were included [18-59]. The flow diagram of the study selection process is shown in Fig. 1.

\section{Study characteristics}

The 42 included studies involved 944 rats. The total animal number in control groups is 470 and the number in acupuncture groups is 474 . 36 studies of all have mentioned specific weight of rats. The rats' weight ranged from 160 to $320 \mathrm{~g}$ in 29 studies. The rats' weight was around $20 \mathrm{~g}$ in 3studies and more than $320 \mathrm{~g}$ in 4 studies. The age of animals was different and mentioned concretely in 20 studies. It ranged from new-born to 24month-old. 1 study used new-born rats; 10 studies used 2-4 months old rats; 6 studies used 6-9 months old rats; 3 studies used aged rats (more than 12 months old). Different subtests of the Morris water maze test were used in these studies: 41 studies with 912 animals reported data as escape latency, 18 studies with 406 animals reported data as frequency of cross platform and 12 studies using 255 animals reported data as time in target quadrant. The rat species included Sprague-Dawley (SD) rats, Wistar rats and AKR rats. Eighteen out of the 42 studies $(42.9 \%, n=377)$ were AD models. Fourteen studies $(33.3 \%, n=309)$ were VD models. And the 10 remaining studies $(23.8 \%)$ used other models. The main characteristics of the 42 studies are shown in Table 1.

\section{Description of acupuncture regime}

Varied acupuncture techniques were used in terms of selection of acupuncture-points, manipulation or stimulation methods (Table 1). The most commonly used acupoints, which have been used by four or more studies, were GV20 (baihui), ST36 (zusanli), GV14 (dazhui), BL23 (shenshu), BL17 (geshu) and CV17 (danzhong). The frequency of acupuncture was mostly once per day. Animals received acupuncture treatment 1 to $30 \mathrm{~min}$ per session. The course of acupuncture treatment ranged from 7 to 60 days. The average duration of acupuncture was 22.4 days $(\mathrm{SD}=10.8) .13$ studies used manual acupuncture, and the rest 29 studies chose electroacupuncture. 5 of 13 studies stated detailed operating methods of manual acupuncture. The operating method of manual acupuncture was mostly twirling reinforcing. 28 of 29 studies stated detailed operating parameters of electroacupuncture and only one ignored related descriptions. 20 of 28 studies used continuous waves. The frequency of continuous wave is from $0.5 \mathrm{~Hz}$ to $150 \mathrm{~Hz}$. The current density of continuous wave is from $0.5 \mathrm{~mA}$ to $30 \mathrm{~mA}$. The rest 8 studies used disperse-dense waves. The frequency of disperse wave is from $1 \mathrm{~Hz}$ to $80 \mathrm{~Hz}$ and the frequency of dense 


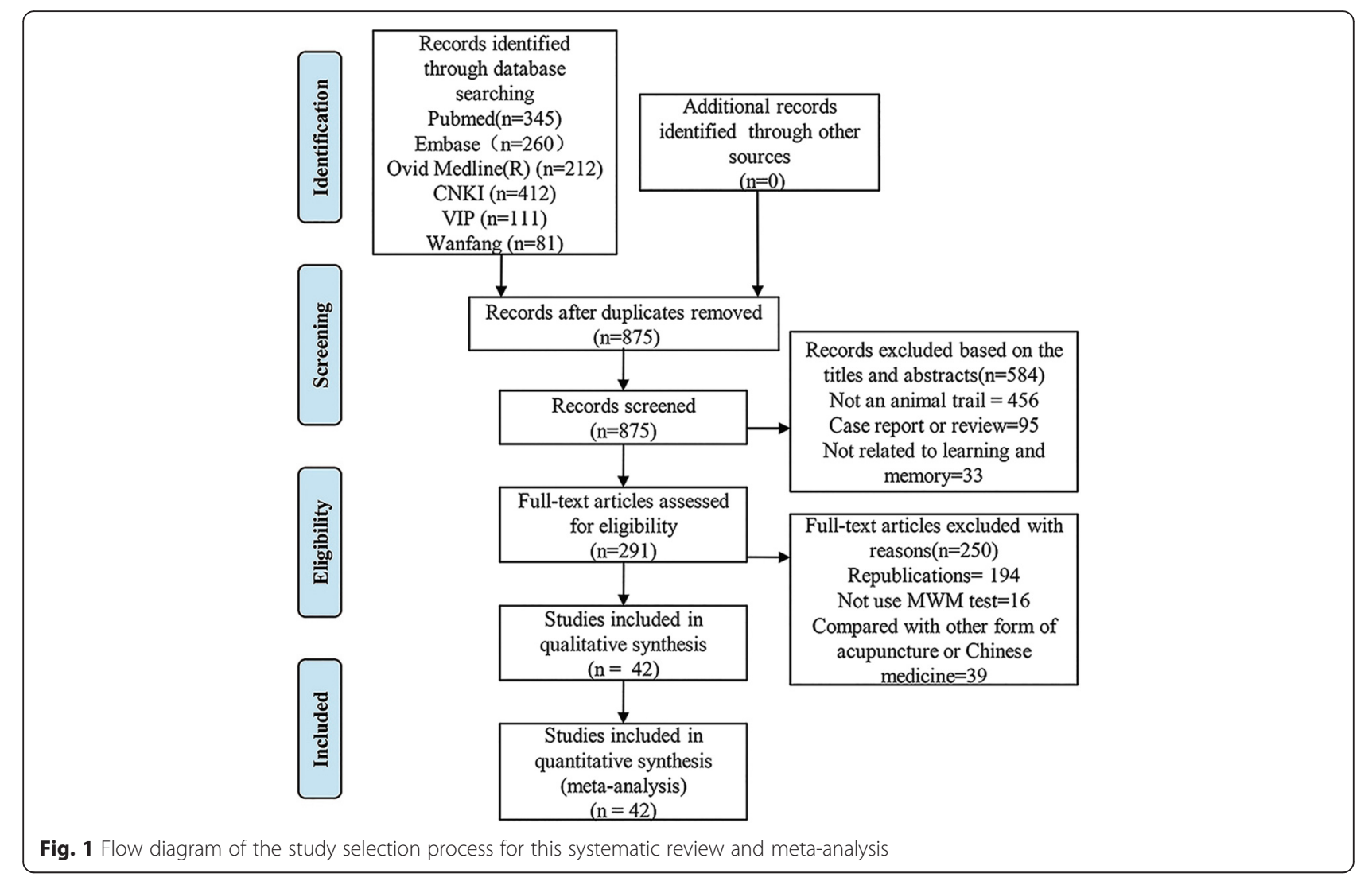

wave is from $15 \mathrm{~Hz}$ to $100 \mathrm{~Hz}$. The current density of continuous wave is from $1 \mathrm{~mA}$ to $3 \mathrm{~mA}$.

\section{Description of control interventions}

Eighteen of included studies used some interventions in control groups (Table 1). Control interventions consisted of western medicine and sham acupuncture. Western medicine was adopted in 9 studies and 9 experiments used sham acupuncture. Medication was administered for similar treatment duration as acupuncture. Types of control medication consisted of donepezil (3 studies), nimodipine (3 studies), fluoxetine (1 study), madopar (1 study) and piracetam (1 study). Puncturing points lateral to acupoints is the way of sham acupuncture in 8 experiments. Not using electroacupuncture apparatus is the other way of sham acupuncture in 1 experiment which adopted electroacupuncture as the intervention way.

\section{Study quality and publication bias}

The score of the study quality was ranged from 2 to 8 out of a total 10 points. Concretely, one study got 2 points; three studies got 3; twelve studies got 4; eleven studies got 5; seven studies got 6; three studies got 7 and five studies got 8 points. Five studies were not published because they were Master's or Ph.D thesis. Twentyseven studies mentioned control of temperature, including control of the room or water temperature. Nineteen studies adopted blinded building of model and seventeen mentioned assessment whether building model was successful. Random allocation to control group or acupuncture group and blinded assessment of outcome were described in 42 and 12 studies. No study reported inducing significant intrinsic neuroprotective activity because of anesthetic. Only one study described the sample size calculation. Fourteen studies reported statement of potential conflict of interests and eleven reported compliance with animal welfare regulations. The study quality and publication bias evaluation are shown in Table 2 .

\section{Effectiveness}

Forty-one studies reported the impact of acupuncture on decreasing escape latency compared with the control group $(p<0.05$ or $p<0.01)$. Twenty-four of them provided detailed data to show significant effectiveness of acupuncture compared with the control group $(n=494$, SMD -3.00, 95 \% CI: $-3.78 \sim-2.23, P<0.00001$; heterogeneity $X^{2}=185.09, \mathrm{I}^{2}=88 \%$, Fig. 2). The remaining seventeen studies did not provide detailed data and just showed the data demonstrated in a graphical form. 
Table 1 Characteristics of the included studies

\begin{tabular}{|c|c|c|c|c|c|c|c|c|}
\hline Study & Species (Nc/Na) & Weight(g) & Age (month) & Model & Acupuncture (acupoints) & Control intervention & Outcome index & $P$ value \\
\hline Bao 2014 [18] & SD Rats (12/12) & $200 \pm 20$ & NR & PTSD (CMS) & $\begin{array}{l}\text { Electroacupuncture } 20 \mathrm{~min} / \mathrm{d} \text { for } 21 \mathrm{~d} \text {, } \\
\text { continuous waves of } 2 \mathrm{~Hz} \text { of frequency } \\
\text { and current density of } 2 \mathrm{~mA} \text { (GV20, } \\
\text { GV29). }\end{array}$ & Fluoxetine & $\begin{array}{l}\text { 1. escape latency } \\
\text { 2. total swimming distance }\end{array}$ & $\begin{array}{l}\text { 1. } P<0.01 \\
\text { 2. } P<0.01\end{array}$ \\
\hline Zeng 2008 [19] & Wistar Rats (10/10) & $250 \pm 10$ & NR & $\mathrm{AD}\left(\mathrm{D}-\mathrm{gal}, \mathrm{NaNO}_{2}\right)$ & $\begin{array}{l}\text { Electroacupuncture } 20 \mathrm{~min} / \mathrm{d} \text { for } 60 \mathrm{~d} \text {, } \\
\text { disperse- dense waves of } 80 / 100 \mathrm{~Hz} \\
\text { of frequency (LI4, LR3). }\end{array}$ & Donepezil & 1. escape latency & 1. $P<0.01$ \\
\hline Chen 2015 [20] & SD Rats (13/14) & $230 \pm 20$ & NR & VD (4-VO) & $\begin{array}{l}\text { Electroacupuncture } 30 \mathrm{~min} / \mathrm{d} \text { for } 7 \mathrm{~d} \text {, } \\
\text { disperse- dense waves of } 1 / 20 \mathrm{~Hz} \text { of } \\
\text { frequency (GV24, GV20). }\end{array}$ & NR & $\begin{array}{l}\text { 1. escape latency } \\
\text { 2.total swimming distance } \\
\text { 3. frequency of cross platform }\end{array}$ & $\begin{array}{l}\text { 1. } P<0.05 \\
\text { 2. } P<0.05 \\
\text { 3. } P<0.05\end{array}$ \\
\hline Chen 2006 [21] & Wistar Rats (34/34) & NR & New-born & HIBD (closed space) & $\begin{array}{l}\text { Manual acupuncture } 20 \mathrm{~min} / \mathrm{d} \text { for } \\
\text { 10d (GV20, GV14). }\end{array}$ & NR & $\begin{array}{l}\text { 1. escape latency } \\
\text { 2. percentage of time in target } \\
\text { quadrant } \\
\text { 3. percentage of swimming } \\
\text { distance in target quadrant }\end{array}$ & $\begin{array}{l}\text { 1.P } P<0.05 \\
\text { 2. } P<0.05 \\
\text { 3. } P<0.05\end{array}$ \\
\hline Dai 2015 [22] & AKR Rats (10/10) & $24.0 \pm 3.5$ & 6 & AD (SAMP8) & $\begin{array}{l}\text { Manual acupuncture } 10 \mathrm{~min} / \mathrm{d} \text { for } 28 \mathrm{~d} \\
\text { (GV20, SP10, BL17, BL23). }\end{array}$ & NR & $\begin{array}{l}\text { 1. escape latency } \\
\text { 2. frequency of cross platform } \\
\text { 3. time in target quadrant }\end{array}$ & $\begin{array}{l}\text { 1. } P<0.05 \\
\text { 2. } P<0.05 \\
\text { 3. } P<0.05\end{array}$ \\
\hline Hou 2013 [23] & SD Rats (10/10) & $220 \pm 20$ & NR & PTSD(SPS) & $\begin{array}{l}\text { Electroacupuncture } 30 \mathrm{~min} / \mathrm{d} \text { for } 7 \mathrm{~d} \text {, } \\
\text { continuous waves of } 2 \mathrm{~Hz} \text { of frequency } \\
\text { and current density of } 1 \mathrm{~mA} \text { (GV20, } \\
\text { ST36). }\end{array}$ & NR & 1. escape latency & 1. $P<0.05$ \\
\hline Gao 2012 [24] & SD Rats $(16 / 16)$ & $400-500$ & $20-24$ & $A D$ (aged rats) & $\begin{array}{l}\text { Electroacupuncture } 30 \mathrm{~min} / \mathrm{d} \text { for } 21 \mathrm{~d} \text {, } \\
\text { disperse- dense waves of } 2 / 100 \mathrm{~Hz} \\
\text { of frequency and intensity of } 2-4 \mathrm{~V} \\
\text { (GV20, KI1). }\end{array}$ & NR & 1. swimming time & 1. $P<0.05$ \\
\hline Huang 2010 [25] & Wistar Rats (10/10) & $200 \pm 20$ & NR & $\mathrm{PD}(6-\mathrm{OH} \mathrm{DA})$ & $\begin{array}{l}\text { Electroacupuncture } 30 \mathrm{~min} / \mathrm{d} \text { for } 24 \mathrm{~d} \text {, } \\
\text { continuous waves of } 100 \mathrm{~Hz} \text { of } \\
\text { frequency and current density of } \\
0.5 \mathrm{~mA}(\mathrm{GV} 16, \mathrm{LR} 3) \text {. }\end{array}$ & Madopar & 1. escape latency & 1. $P<0.01$ \\
\hline Ji 2011 [26] & SD Rats $(10 / 10)$ & $220-260$ & NR & VD (4-VO) & $\begin{array}{l}\text { Manual acupuncture } 30 \mathrm{~min} / \mathrm{d} \text { for } 30 \mathrm{~d} \\
\text { (GV20, CV17, CV6, BL17, SP6). }\end{array}$ & NR & $\begin{array}{l}\text { 1. escape latency } \\
\text { 2. frequency of cross platform }\end{array}$ & $\begin{array}{l}\text { 1. } P<0.01 \\
\text { 2. } P<0.01\end{array}$ \\
\hline Jia 2011 [27] & SD Rats $(20 / 20)$ & $220 \pm 20$ & NR & VD (2-VO) & $\begin{array}{l}\text { Electroacupuncture } 20 \mathrm{~min} / \mathrm{d} \text { for } 14 \mathrm{~d} \text {, } \\
\text { continuous waves of } 2 \mathrm{~Hz} \text { of frequency } \\
\text { and intensity of } 3 \mathrm{~V}(\mathrm{GV} 2 \mathrm{O}, \mathrm{GV} 14) \text {. }\end{array}$ & NR & $\begin{array}{l}\text { 1. escape latency } \\
\text { 2. frequency of cross platform }\end{array}$ & $\begin{array}{l}\text { 1. } P<0.05 \\
\text { 2. } P<0.05\end{array}$ \\
\hline Wang 2012 [28] & Wistar Rats (10/10) & $300 \pm 10$ & NR & $A D(S T Z)$ & $\begin{array}{l}\text { Electroacupuncture for 28d (GV20, } \\
\text { GV14, ST36). }\end{array}$ & NR & $\begin{array}{l}\text { 1. escape latency } \\
\text { 2. frequency of cross platform } \\
\text { 3. time in target quadrant }\end{array}$ & $\begin{array}{l}\text { 1. } P<0.05 \\
\text { 2. } P<0.05 \\
\text { 3. } P<0.05\end{array}$ \\
\hline Lin 2008 [29] & SD Rats (10/10) & $620 \pm 80$ & 12 & VD (2-VO) & $\begin{array}{l}\text { Electroacupuncture } 20 \mathrm{~min} / \mathrm{d} \text { for } 30 \mathrm{~d}, \\
\text { continuous waves of } 2 \mathrm{~Hz} \text { of frequency } \\
\text { and current density of } 1-2 \mathrm{~mA} \text { (GV20, } \\
\text { GV14,BL23). }\end{array}$ & Sham acupuncture & $\begin{array}{l}\text { 1. escape latency } \\
\text { 2. time in target quadrant }\end{array}$ & $\begin{array}{l}\text { 1. } P<0.05 \\
\text { 2. } P<0.05\end{array}$ \\
\hline
\end{tabular}


Table 1 Characteristics of the included studies (Continued)

\begin{tabular}{|c|c|c|c|c|c|c|c|c|}
\hline Luo 2007 [30] & SD Rats (14/14) & $200 \pm 20$ & NR & VD (4-VO) & $\begin{array}{l}\text { Electroacupuncture } 20 \mathrm{~min} / \mathrm{d} \text { for } 15 \mathrm{~d} \text {, } \\
\text { continuous waves of } 150 \mathrm{~Hz} \text { of } \\
\text { frequency and current density of } \\
1 \mathrm{~mA} \text { (GV20, BL17, BL20, BL23). }\end{array}$ & Nimodipine & 1. escape latency & 1. $P<0.01$ \\
\hline Ma 2009 [31] & Wistar Rats (13/13) & $200-250$ & NR & Diabete (STZ) & $\begin{array}{l}\text { Electroacupuncture } 15 \mathrm{~min} / \mathrm{d} \text { for } 14 \mathrm{~d} \text {, } \\
\text { continuous waves of } 0.5 \mathrm{~Hz} \text { of } \\
\text { frequency and current density of } \\
30 \mathrm{~mA} \text { (GV20, GV14). }\end{array}$ & NR & 1. escape latency & 1. $P<0.05$ \\
\hline Zhang 2014 [32] & AKR Rats (10/10) & NR & 4 & AD (SAMP8) & $\begin{array}{l}\text { Manual acupuncture } 30 \mathrm{~min} / \mathrm{d} \text { for } 30 \mathrm{~d} \text {, } \\
\text { twisting } 10 \mathrm{~s} \text { a time (GV20,ST36). }\end{array}$ & NR & $\begin{array}{l}\text { 1. escape latency } \\
\text { 2. frequency of cross platform } \\
\text { 3. time in target quadrant }\end{array}$ & $\begin{array}{l}\text { 1. } P<0.05 \\
\text { 2. } P<0.05 \\
\text { 3. } P<0.05\end{array}$ \\
\hline Niu 2009 [33] & SD Rats (10/10) & $300 \pm 20$ & NR & VD (4-VO) & $\begin{array}{l}\text { Electroacupuncture } 10 \mathrm{~min} / \mathrm{d} \text { for } 42 \mathrm{~d} \text {, } \\
\text { disperse- dense waves of } 80 / 100 \mathrm{~Hz} \\
\text { of frequency and current density of } \\
1-3 \mathrm{~mA}(\mathrm{GV} 29, \mathrm{LI} 20) \text {. }\end{array}$ & NR & $\begin{array}{l}\text { 1. escape latency } \\
\text { 2. frequency of cross platform }\end{array}$ & $\begin{array}{l}\text { 1. } P<0.01 \\
\text { 2. } P<0.01\end{array}$ \\
\hline Su 2013 [34] & SD Rats (12/12) & $200-250$ & NR & $A D(D-g a l, A \beta 1-42)$ & $\begin{array}{l}\text { Electroacupuncture } 15 \mathrm{~min} / \mathrm{d} \text { for } 28 \mathrm{~d} \text {, } \\
\text { continuous waves of } 35 \mathrm{~Hz} \text { of } \\
\text { frequency and intensity of } 2 \mathrm{~V}(\mathrm{GV} 20 \text {, } \\
\mathrm{KI} 3, \text { ST36). }\end{array}$ & NR & $\begin{array}{l}\text { 1. escape latency } \\
\text { 2. frequency of cross platform } \\
\text { 3. time in target quadrant }\end{array}$ & $\begin{array}{l}\text { 1. } P<0.05 \\
\text { 2. } P<0.05 \\
\text { 3. } P<0.05\end{array}$ \\
\hline Tan 2014 [35] & Wistar Rats (8/8) & $250 \pm 50$ & 2 & VD (MCAO) & $\begin{array}{l}\text { Electroacupuncture } 15 \mathrm{~min} / \mathrm{d} \text { for } 21 \mathrm{~d} \text {, } \\
\text { continuous waves of } 16 \mathrm{~Hz} \text { of } \\
\text { frequency and current density of } \\
1 \mathrm{~mA} \text { (GV20, GV14). }\end{array}$ & NR & $\begin{array}{l}\text { 1. escape latency } \\
\text { 2. frequency of cross platform } \\
\text { 3. percentage of time in target } \\
\text { quadrant }\end{array}$ & $\begin{array}{l}\text { 1. } P<0.05 \\
\text { 2. } P<0.05 \\
\text { 3. } P<0.05\end{array}$ \\
\hline Tang 2014 [36] & SD Rats (10/10) & $160-200$ & 3 & ovx & $\begin{array}{l}\text { Electroacupuncture } 20 \mathrm{~min} / \mathrm{d} \text { for } 45 \mathrm{~d} \text {, } \\
\text { continuous waves of } 3-4 \mathrm{~Hz} \text { of } \\
\text { frequency and current density of } \\
4-5 \mathrm{~mA} \text { (ST36, BL23). }\end{array}$ & Sham acupuncture & $\begin{array}{l}\text { 1. escape latency } \\
\text { 2. frequency of cross platform }\end{array}$ & $\begin{array}{l}\text { 1. } P<0.01 \\
\text { 2. } P<0.01\end{array}$ \\
\hline Wang 2013 [37] & SD Rats $(10 / 10)$ & $200 \pm 20$ & NR & $\mathrm{AD}\left(\mathrm{D}-\mathrm{gal}, \mathrm{NaNO}_{2}\right)$ & $\begin{array}{l}\text { Manual acupuncture } 10 \mathrm{~min} / \mathrm{d} \text { for } 30 \mathrm{~d} \\
(\text { LI4, LR3, ST36). }\end{array}$ & Donepezil & $\begin{array}{l}\text { 1. escape latency } \\
\text { 2. frequency of cross platform }\end{array}$ & $\begin{array}{l}\text { 1. } P<0.01 \\
\text { 2. } P<0.01\end{array}$ \\
\hline Wang 2009 [38] & SD Rats (12/13) & $240 \pm 20$ & 3 & VD (2-VO) & $\begin{array}{l}\text { Manual acupuncture } 10 \mathrm{~min} / \mathrm{d} \text { for } 30 \mathrm{~d} \\
\text { (GV20, BL17, CV6, SP6, CV17). }\end{array}$ & Piracetam & $\begin{array}{l}\text { 1. escape latency } \\
\text { 2. frequency of cross platform }\end{array}$ & $\begin{array}{l}\text { 1. } P<0.01 \\
\text { 2. } P<0.05\end{array}$ \\
\hline Hong 2014 [39] & Wistar Rats (10/10) & $300-350$ & NR & Autism (VPA) & $\begin{array}{l}\text { Manual acupuncture } 1 \mathrm{~min} / \mathrm{d} \text { for } 30 \mathrm{~d} \\
\text { (GV1). }\end{array}$ & Sham acupuncture & $\begin{array}{l}\text { 1. escape latency } \\
\text { 2. average speed }\end{array}$ & $\begin{array}{l}\text { 1. } P<0.05 \\
\text { 2. } P<0.05\end{array}$ \\
\hline Xu 2006 [40] & SD Rats (13/14) & $200-220$ & 2 & VD (4-VO) & $\begin{array}{l}\text { Electroacupuncture } 20 \mathrm{~min} / \mathrm{d} \text { for } 20 \mathrm{~d} \text {, } \\
\text { continuous waves of } 150 \mathrm{~Hz} \text { of } \\
\text { frequency and current density of } \\
20 \mathrm{~mA} \text { (GV20, GV14). }\end{array}$ & Nimodipine & $\begin{array}{l}\text { 1. escape latency } \\
\text { 2. frequency of cross platform }\end{array}$ & $\begin{array}{l}\text { 1. } P<0.01 \\
\text { 2. } P<0.01\end{array}$ \\
\hline Xu 2007 [41] & SD Rats $(8 / 10)$ & $180-200$ & NR & AD (D-gal) & $\begin{array}{l}\text { Electroacupuncture } 20 \mathrm{~min} / \mathrm{d} \text { for } 21 \mathrm{~d} \text {, } \\
\text { continuous waves of } 3 \mathrm{~Hz} \text { of } \\
\text { frequency and current density of } \\
1 \mathrm{~mA} \text { (GV20, ST36). }\end{array}$ & NR & $\begin{array}{l}\text { 1. escape latency } \\
\text { 2. percentage of swimming } \\
\text { distance in target quadrant } \\
\text { 3. percentage of time in target } \\
\text { quadrant }\end{array}$ & $\begin{array}{l}\text { 1. } P<0.01 \\
\text { 2. } P<0.01 \\
\text { 3. } P<0.01\end{array}$ \\
\hline
\end{tabular}


Table 1 Characteristics of the included studies (Continued)

\begin{tabular}{|c|c|c|c|c|c|c|c|c|}
\hline Yi 2014 [42] & SD Rats (12/12) & $200 \pm 34$ & 4 & $A D(A \beta 25-35)$ & $\begin{array}{l}\text { Electroacupuncture } 30 \mathrm{~min} / \mathrm{d} \text { for } 12 \mathrm{~d} \text {, } \\
\text { disperse- dense waves of } 2 / 30 \mathrm{~Hz} \text { of } \\
\text { frequency and current density of } \\
1 \mathrm{~mA}(\mathrm{GV} 29, \mathrm{LI} 20) \text {. }\end{array}$ & NR & $\begin{array}{l}\text { 1. escape latency } \\
\text { 2. frequency of cross platform } \\
\text { 3. time in target quadrant }\end{array}$ & $\begin{array}{l}\text { 1. } P<0.05 \\
\text { 2. } P<0.05 \\
\text { 3. } P<0.05\end{array}$ \\
\hline Yu 2014 [43] & Wistar Rats (10/10) & $200-250$ & NR & WD (CuSO4) & $\begin{array}{l}\text { Electroacupuncture } 15 \mathrm{~min} / \mathrm{d} \text { for } 7 \mathrm{~d} \text {, } \\
\text { continuous waves of } 2 \mathrm{~Hz} \text { of frequency } \\
\text { and current density of } 1 \mathrm{~mA} \text { (HT7). }\end{array}$ & NR & 1. escape latency & 1. $P<0.01$ \\
\hline Feng 2013 [44] & AKR Rats (10/9) & $29-35$ & 9 & AD (SAMP8) & $\begin{array}{l}\text { Manual acupuncture } 20 \mathrm{~min} / \mathrm{d} \text { for } 28 \mathrm{~d} \text {, } \\
\text { twisting } 2.5 \text { times/s for } 60 \mathrm{~s}(\mathrm{GV} 20, \mathrm{KI}) \text {. }\end{array}$ & NR & $\begin{array}{l}\text { 1. escape latency } \\
\text { 2. frequency of cross platform }\end{array}$ & $\begin{array}{l}\text { 1. } P<0.05 \\
\text { 2. } P<0.05\end{array}$ \\
\hline Li 2013 [45] & Wistar Rats (10/10) & $200-250$ & 4 & $A D(S T Z)$ & $\begin{array}{l}\text { Electroacupuncture for } 28 \mathrm{~d} \text {, continuous } \\
\text { waves of } 30 \mathrm{~Hz} \text { of frequency and } \\
\text { intensity of } 2 \vee(B L 23, \mathrm{KI} 3, \text { ST36, } \\
\text { GV20, GV14). }\end{array}$ & Donepezil & $\begin{array}{l}\text { 1. escape latency } \\
\text { 2. frequency of cross platform }\end{array}$ & $\begin{array}{l}\text { 1. } P<0.05 \\
\text { 2. } P<0.05\end{array}$ \\
\hline Wang 2013 [46] & AKR Rats (10/10) & NR & 8 & AD (SAMP8) & $\begin{array}{l}\text { Manual acupuncture for 15d (CV17, } \\
\text { CV12, CV6, SP10,ST36). }\end{array}$ & Sham acupuncture & $\begin{array}{l}\text { 1. escape latency } \\
\text { 2. time in target quadrant }\end{array}$ & $\begin{array}{l}\text { 1. } P<0.01 \\
\text { 2. } P<0.01\end{array}$ \\
\hline Zheng 2009 [47] & Wistar Rats (8/7) & $212 \pm 15$ & 2 & VD (2-VO) & $\begin{array}{l}\text { Electroacupuncture for } 28 \mathrm{~d} \text {, continuous } \\
\text { waves of } 2 \mathrm{~Hz} \text { of frequency }(\mathrm{GV} 20, \mathrm{~K} / 3) \text {. }\end{array}$ & NR & 1. escape latency & 1. $P<0.05$ \\
\hline Li 2012 [48] & AKR Rats (15/15) & NR & 7.5 & AD (SAMP8) & $\begin{array}{l}\text { Manual acupuncture for 15d, twisting } \\
2 \text { times/s for } 30 \text { s (CV17, CV12, CV6, } \\
\text { SP10, ST36). }\end{array}$ & Sham acupuncture & $\begin{array}{l}\text { 1. escape latency } \\
\text { 2. time in target quadrant }\end{array}$ & $\begin{array}{l}\text { 1. } P<0.05 \\
\text { 2. } P<0.05\end{array}$ \\
\hline Li 2014 [49] & C57BL/6 Rats (6/6) & NR & 2 & $A D(A P P / P S 1)$ & $\begin{array}{l}\text { Electroacupuncture } 30 \mathrm{~min} / \mathrm{d} \text { for } 20 \mathrm{~d} \text {, } \\
\text { disperse- dense waves of } 2 / 15 \mathrm{~Hz} \text { of } \\
\text { frequency and current density of } \\
1 \mathrm{~mA} \text { (GV20). }\end{array}$ & NR & 1. escape latency & 1. $P<0.05$ \\
\hline Lee 2014 [50] & SD Rats (7/7) & $220-240$ & NR & $\mathrm{AD}(\mathrm{SCO})$ & Manual acupuncture for 15d (GV20). & Sham acupuncture & $\begin{array}{l}\text { 1. escape latency } \\
\text { 2. swimming speed } \\
\text { 3. percentages of time in a } \\
\text { probe trial }\end{array}$ & $\begin{array}{l}\text { 1. } P<0.05 \\
\text { 2. } P<0.05 \\
\text { 3. } P<0.05\end{array}$ \\
\hline Zhu 2013 [51] & SD Rats (6/6) & $432 \pm 30$ & 12 & VD (2-VO) & $\begin{array}{l}\text { Electroacupuncture } 20 \mathrm{~min} / \mathrm{d} \text { for } 30 \mathrm{~d} \text {, } \\
\text { continuous waves of } 4 \mathrm{~Hz} \text { of frequency } \\
\text { and current density of } 2 \mathrm{~mA} \text { (GV20, } \\
\text { GV14, BL23). }\end{array}$ & NR & 1. escape latency & 1. $P<0.05$ \\
\hline Lu 2014 [52] & SD Rats (8/8) & $200-250$ & NR & Ethanol & $\begin{array}{l}\text { Electroacupuncture } 20 \mathrm{~min} / \mathrm{d} \text { for } 30 \mathrm{~d} \text {, } \\
\text { continuous waves of } 2 \mathrm{~Hz} \text { of frequency } \\
\text { and current density of } 1.5-2 \mathrm{~mA} \text { (ST36). }\end{array}$ & Sham acupuncture & $\begin{array}{l}\text { 1. escape latency } \\
\text { 2. time in target quadrant }\end{array}$ & $\begin{array}{l}\text { 1. } P<0.05 \\
\text { 2. } P<0.05\end{array}$ \\
\hline Li 2012 [53] & SD Rats (10/10) & $250 \pm 30$ & 3 & VD (MCAO) & $\begin{array}{l}\text { Electroacupuncture } 30 \mathrm{~min} / \mathrm{d} \text { for } 14 \mathrm{~d} \text {, } \\
\text { disperse- dense waves of } 2 / 30 \mathrm{~Hz} \text { of } \\
\text { frequency and current density of } \\
2 \mathrm{~mA} \text { (GV20, GV14). }\end{array}$ & NR & 1. escape latency & 1. $P<0.01$ \\
\hline Guo 2015 [54] & SD Rats (10/10) & $250-300$ & NR & $A D(A \beta 1-40)$ & $\begin{array}{l}\text { Electroacupuncture } 30 \mathrm{~min} / \mathrm{d} \text { for } 24 \mathrm{~d} \text {, } \\
\text { continuous waves of } 20 \mathrm{~Hz} \text { of } \\
\text { frequency and current density of less } \\
\text { than } 2 \mathrm{~mA} \text { (GV20, BL23). }\end{array}$ & Sham acupuncture & $\begin{array}{l}\text { 1. escape latency } \\
\text { 2. time in target quadrant } \\
\text { 3. frequency of cross platform }\end{array}$ & $\begin{array}{l}\text { 1. } P<0.01 \\
\text { 2. } P<0.01 \\
\text { 3. } P<0.01\end{array}$ \\
\hline
\end{tabular}


Table 1 Characteristics of the included studies (Continued)

\begin{tabular}{|c|c|c|c|c|c|c|c|c|}
\hline Jiang 2015 [55] & AKR Rats $(10 / 10)$ & NR & 7.5 & AD (SAMP8) & $\begin{array}{l}\text { Electroacupuncture for } 14 \mathrm{~d} \text {, continuous } \\
\text { waves of } 2 \mathrm{~Hz} \text { of frequency, current } \\
\text { density of } 0.6 \mathrm{~mA} \text { and intensity of } \\
2 \vee(\mathrm{GV} 20, \mathrm{GV} 26, \mathrm{GV} 29) \text {. }\end{array}$ & NR & $\begin{array}{l}\text { 1. escape latency } \\
\text { 2. percentages of time in target } \\
\text { quadrant }\end{array}$ & $\begin{array}{l}\text { 1. } P<0.01 \\
\text { 2. } P<0.01\end{array}$ \\
\hline Shao 2008 [56] & SD Rats (8/9) & $180-220$ & $N R$ & VD (4-VO) & $\begin{array}{l}\text { Electroacupuncture } 20 \mathrm{~min} / \mathrm{d} \text { for } 15 \mathrm{~d} \text {, } \\
\text { continuous waves of } 150 \mathrm{~Hz} \text { of } \\
\text { frequency and current density of } \\
1-2 \mathrm{~mA} \text { (GV20, BL17, BL20, BL23). }\end{array}$ & Nimodipine & $\begin{array}{l}\text { 1. escape latency } \\
\text { 2. time in target quadrant }\end{array}$ & $\begin{array}{l}\text { 1. } P<0.01 \\
\text { 2. } P<0.01\end{array}$ \\
\hline Liu 2013 [57] & SD Rats (12/12) & $200 \pm 20$ & NR & CFS & $\begin{array}{l}\text { Manual acupuncture } 20 \mathrm{~min} / \mathrm{d} \text { for } 21 \mathrm{~d} \text {, } \\
\text { twirling reinforcing (ST36). }\end{array}$ & NR & $\begin{array}{l}\text { 1. escape latency } \\
\text { 2. frequency of cross platform }\end{array}$ & $\begin{array}{l}\text { 1. } P<0.05 \\
\text { 2. } P<0.05\end{array}$ \\
\hline Li 2015 [58] & Wistar Rats (11/11) & $320-360$ & NR & VD(micro- emboli) & $\begin{array}{l}\text { Manual acupuncture for 12d, twisting } \\
2 \text { times/s for } 30 \mathrm{~s} \text { (ST36). }\end{array}$ & Sham acupuncture & 1. escape latency & 1. $P<0.01$ \\
\hline Lu 2008 [59] & AKR Rats (12/12) & $20 \pm 2$ & 8 & AD (SAMP8) & $\begin{array}{l}\text { Electroacupuncture } 20 \mathrm{~min} / \mathrm{d} \text { for } 7 \mathrm{~d} \text {, } \\
\text { disperse- dense waves of } 2 / 100 \mathrm{~Hz} \\
\text { of frequency and intensity of } 2-4 \mathrm{~V} \\
(\mathrm{GV} 20, \mathrm{KI} 1) \text {. }\end{array}$ & NR & $\begin{array}{l}\text { 1. escape latency } \\
\text { 2. time in target quadrant }\end{array}$ & $\begin{array}{l}\text { 1. } P<0.05 \\
\text { 2. } P<0.05\end{array}$ \\
\hline
\end{tabular}

$N c$ animal number in control group, Na animal number in acupuncture group, PTSD post-traumatic stress disorder, CMS chronic mild stimulation, NR not report, $A D$ Alzheimer's disease, $V D$ vascular dementia, 4-VO

4- vessel occlusion, SPS single prolonged stress, PD Parkinson's disease, 6-OHDA 6-OH-dopamine, STZ streptozotocin, CFS chronic fatigue syndrome, MCAO middle cerebral artery occlusion, OVX ovariectomy, VPA sodium valproate, WD Wilson disease 
Table 2 Risk of bias of included studies

\begin{tabular}{|c|c|c|c|c|c|c|c|c|c|c|c|}
\hline Study & (1) & (2) & (3) & (4) & (5) & (6) & (7) & (8) & (9) & (10) & Total \\
\hline Bao 2014 [18] & $\sqrt{ }$ & $\sqrt{ }$ & $\sqrt{ }$ & & $\sqrt{ }$ & $\sqrt{ }$ & $\sqrt{ }$ & & & & 6 \\
\hline Zeng 2008 [19] & $\sqrt{ }$ & $\sqrt{ }$ & $\sqrt{ }$ & & & & $\sqrt{ }$ & & & & 4 \\
\hline Chen 2015 [20] & $\sqrt{ }$ & $\sqrt{ }$ & $\sqrt{ }$ & & $\sqrt{ }$ & $\sqrt{ }$ & $\sqrt{ }$ & $\sqrt{ }$ & $\sqrt{ }$ & & 8 \\
\hline Chen 2006 [21] & & $\sqrt{ }$ & $\sqrt{ }$ & & $\sqrt{ }$ & & $\sqrt{ }$ & & & $\sqrt{ }$ & 5 \\
\hline Dai 2015 [22] & $\sqrt{ }$ & $\sqrt{ }$ & $\sqrt{ }$ & $\sqrt{ }$ & & $\sqrt{ }$ & & & & & 5 \\
\hline Hou 2013 [23] & $\sqrt{ }$ & $\sqrt{ }$ & $\sqrt{ }$ & & $\sqrt{ }$ & & & & $\sqrt{ }$ & & 5 \\
\hline Gao 2012 [24] & & $\sqrt{ }$ & $\sqrt{ }$ & & $\sqrt{ }$ & & & & & $\sqrt{ }$ & 4 \\
\hline Huang 2010 [25] & $\sqrt{ }$ & & $\sqrt{ }$ & & $\sqrt{ }$ & & $\sqrt{ }$ & & & & 4 \\
\hline Ji 2011 [26] & $\sqrt{ }$ & $\sqrt{ }$ & $\sqrt{ }$ & & & & $\sqrt{ }$ & & & & 4 \\
\hline Jia 2011 [27] & & & $\sqrt{ }$ & & $\sqrt{ }$ & & $\sqrt{ }$ & & & $\sqrt{ }$ & 4 \\
\hline Wang 2012 [28] & $\sqrt{ }$ & $\sqrt{ }$ & $\sqrt{ }$ & $\sqrt{ }$ & $\sqrt{ }$ & & $\sqrt{ }$ & & & & 6 \\
\hline Lin 2008 [29] & $\sqrt{ }$ & & $\sqrt{ }$ & & $\sqrt{ }$ & & $\sqrt{ }$ & & & & 4 \\
\hline Luo 2007 [30] & $\sqrt{ }$ & & $\sqrt{ }$ & & & & & & & & 2 \\
\hline Ma 2009 [31] & $\sqrt{ }$ & & $\sqrt{ }$ & $\sqrt{ }$ & $\sqrt{ }$ & & $\sqrt{ }$ & & & & 5 \\
\hline Zhang 2014 [32] & $\sqrt{ }$ & & $\sqrt{ }$ & $\sqrt{ }$ & $\sqrt{ }$ & & $\sqrt{ }$ & & & & 5 \\
\hline Niu 2009 [33] & $\sqrt{ }$ & $\sqrt{ }$ & $\sqrt{ }$ & & & & $\sqrt{ }$ & & & & 4 \\
\hline Su 2013 [34] & $\sqrt{ }$ & & $\sqrt{ }$ & & & & $\sqrt{ }$ & & & & 3 \\
\hline Tan 2014 [35] & $\sqrt{ }$ & $\sqrt{ }$ & $\sqrt{ }$ & $\sqrt{ }$ & $\sqrt{ }$ & $\sqrt{ }$ & $\sqrt{ }$ & & & & 7 \\
\hline Tang 2014 [36] & $\sqrt{ }$ & & $\sqrt{ }$ & & & & $\sqrt{ }$ & & & & 3 \\
\hline Wang 2013 [37] & & $\sqrt{ }$ & $\sqrt{ }$ & & & & $\sqrt{ }$ & & & $\sqrt{ }$ & 4 \\
\hline Wang 2009 [38] & & & $\sqrt{ }$ & & $\sqrt{ }$ & & $\sqrt{ }$ & & & $\sqrt{ }$ & 4 \\
\hline Hong 2014 [39] & $\sqrt{ }$ & $\sqrt{ }$ & $\sqrt{ }$ & $\sqrt{ }$ & $\sqrt{ }$ & & $\sqrt{ }$ & & & & 6 \\
\hline Xu 2006 [40] & $\sqrt{ }$ & & $\sqrt{ }$ & $\sqrt{ }$ & & & $\sqrt{ }$ & & & & 4 \\
\hline Xu 2007 [41] & $\sqrt{ }$ & $\sqrt{ }$ & $\sqrt{ }$ & & & $\sqrt{ }$ & $\sqrt{ }$ & & & & 5 \\
\hline Yi 2014 [42] & $\sqrt{ }$ & & $\sqrt{ }$ & $\sqrt{ }$ & $\sqrt{ }$ & $\sqrt{ }$ & $\sqrt{ }$ & & & & 6 \\
\hline Yu 2014 [43] & $\sqrt{ }$ & $\sqrt{ }$ & $\sqrt{ }$ & & & $\sqrt{ }$ & $\sqrt{ }$ & & & & 5 \\
\hline Feng 2013 [44] & $\sqrt{ }$ & $\sqrt{ }$ & $\sqrt{ }$ & $\sqrt{ }$ & & & $\sqrt{ }$ & & & & 5 \\
\hline Li 2013 [45] & $\sqrt{ }$ & & $\sqrt{ }$ & $\sqrt{ }$ & & & $\sqrt{ }$ & & & & 4 \\
\hline Wang 2013 [46] & $\sqrt{ }$ & $\sqrt{ }$ & $\sqrt{ }$ & $\sqrt{ }$ & & $\sqrt{ }$ & $\sqrt{ }$ & & & & 6 \\
\hline Zheng 2009 [47] & $\sqrt{ }$ & & $\sqrt{ }$ & & & & $\sqrt{ }$ & & & & 3 \\
\hline Li 2012 [48] & $\sqrt{ }$ & $\sqrt{ }$ & $\sqrt{ }$ & $\sqrt{ }$ & & $\sqrt{ }$ & $\sqrt{ }$ & & $\sqrt{ }$ & $\sqrt{ }$ & 8 \\
\hline Li 2014 [49] & $\sqrt{ }$ & $\sqrt{ }$ & $\sqrt{ }$ & $\sqrt{ }$ & & & $\sqrt{ }$ & & $\sqrt{ }$ & $\sqrt{ }$ & 7 \\
\hline Lee 2014 [50] & $\sqrt{ }$ & $\sqrt{ }$ & $\sqrt{ }$ & $\sqrt{ }$ & & $\sqrt{ }$ & $\sqrt{ }$ & & $\sqrt{ }$ & $\sqrt{ }$ & 8 \\
\hline Zhu 2013 [51] & $\sqrt{ }$ & $\sqrt{ }$ & $\sqrt{ }$ & $\sqrt{ }$ & & & $\sqrt{ }$ & & & $\sqrt{ }$ & 6 \\
\hline Lu 2014 [52] & $\sqrt{ }$ & $\sqrt{ }$ & $\sqrt{ }$ & & $\sqrt{ }$ & $\sqrt{ }$ & $\sqrt{ }$ & & $\sqrt{ }$ & $\sqrt{ }$ & 8 \\
\hline Li 2012 [53] & $\sqrt{ }$ & & $\sqrt{ }$ & & $\sqrt{ }$ & & $\sqrt{ }$ & & $\sqrt{ }$ & & 5 \\
\hline Guo 2015 [54] & $\sqrt{ }$ & $\sqrt{ }$ & $\sqrt{ }$ & & & & $\sqrt{ }$ & & $\sqrt{ }$ & $\sqrt{ }$ & 6 \\
\hline Jiang 2015 [55] & $\sqrt{ }$ & $\sqrt{ }$ & $\sqrt{ }$ & $\sqrt{ }$ & & & $\sqrt{ }$ & & $\sqrt{ }$ & $\sqrt{ }$ & 7 \\
\hline Shao 2008 [56] & $\sqrt{ }$ & $\sqrt{ }$ & $\sqrt{ }$ & $\sqrt{ }$ & & & & & & $\sqrt{ }$ & 5 \\
\hline Liu 2013 [57] & $\sqrt{ }$ & $\sqrt{ }$ & $\sqrt{ }$ & & & & $\sqrt{ }$ & & $\sqrt{ }$ & & 5 \\
\hline Li 2015 [58] & $\sqrt{ }$ & $\sqrt{ }$ & $\sqrt{ }$ & $\sqrt{ }$ & & $\sqrt{ }$ & $\sqrt{ }$ & & $\sqrt{ }$ & $\sqrt{ }$ & 8 \\
\hline Lu 2008 [59] & $\sqrt{ }$ & & $\sqrt{ }$ & $\sqrt{ }$ & & & $\sqrt{ }$ & & & & 4 \\
\hline
\end{tabular}

(1) publication in a peer-reviewed journal; (2) statements describing control of temperature; (3) random allocation to treatment or control; (4) blinded building of model; (5) assessment whether building model is successful; (6) blinded assessment of outcome;(7) use of anesthetic without significant intrinsic neuroprotective activity; (8) sample size calculation; (9) compliance with animal welfare regulations; (10) declared any potential conflict of interest
Eighteen studies reported the impact of acupuncture on increasing frequency of cross platform compared with the control group $(p<0.05$ or $p<0.01)$. Fourteen of them provided detailed data to show significant effectiveness of acupuncture compared with the control group ( $n=317$, SMD 2.57, $95 \%$ CI: $1.92 \sim 3.22, P<$ 0.00001; heterogeneity $\chi^{2}=52.81, \mathrm{I}^{2}=75 \%$, Fig. 3). The remaining four studies just showed the data demonstrated in the graphical form and failed for meta-analysis.

Eleven studies reported the impact of acupuncture on increasing time in target quadrant compared with the control group $(p<0.05$ or $p<0.01)$. Seven of them provided detailed data to show significant effectiveness of acupuncture compared with the control group $(n=149$, SMD 2.00, $95 \%$ CI: $1.10 \sim 2.91, P<0.00001$; heterogeneity $\chi^{2}=28.18, \mathrm{I}^{2}=79 \%$, Fig. 4). The remaining five studies just showed the data demonstrated in the graphical form.

\section{Signaling pathways}

Several different signaling pathways were investigated to gain a better understanding of the underlying mechanism of acupuncture in the amelioration of learning and memory impairment. 39 of 42 included studies got detailed descriptions about possible mechanisms. It can be found that reduction nerve apoptosis and necrosis and suppression of oxidative stress are the main signaling pathways. A summary of proposed mechanism is shown in Table 3.

\section{Assessment of bias}

The funnel plot was approximately symmetric for the effect of acupuncture on escape latency, frequency of cross platform and time in target quadrant (Fig. 5). Nevertheless, publication bias was still considered to be potential.

\section{Affecting factors of outcome indexes}

In the subgroup analysis of escape latency, the efficacy of manual acupuncture was better than electroacupuncture (SMD -4.09, $95 \%$ CI: $-6.24 \sim-1.95$, Fig. 6a). Compared with published articles, unpublished articles showed more obvious changes of escape latency caused by acupuncture treatment (SMD -6.89, $95 \%$ CI: $-8.68 \sim-5.29$, Fig. $6 \mathrm{~b})$. According to different varieties of experimental animals, SD rats were more sensitive to acupuncture than Wister rats and AKR rats (SMD $-3.83,95 \% \mathrm{CI}:-5.21 \sim-2.45$, Fig. $6 \mathrm{c}$ ). AD and $\mathrm{VD}$ are the common diseases causing learning and memory impairment. After treatment, escape latency was further improved in $\mathrm{AD}$ animal model (SMD -3.23, $95 \%$ CI: $-4.48 \sim-1.99$, Fig. 6d). There are many ways making $\mathrm{AD}$ and $\mathrm{VD}$ animal models. 


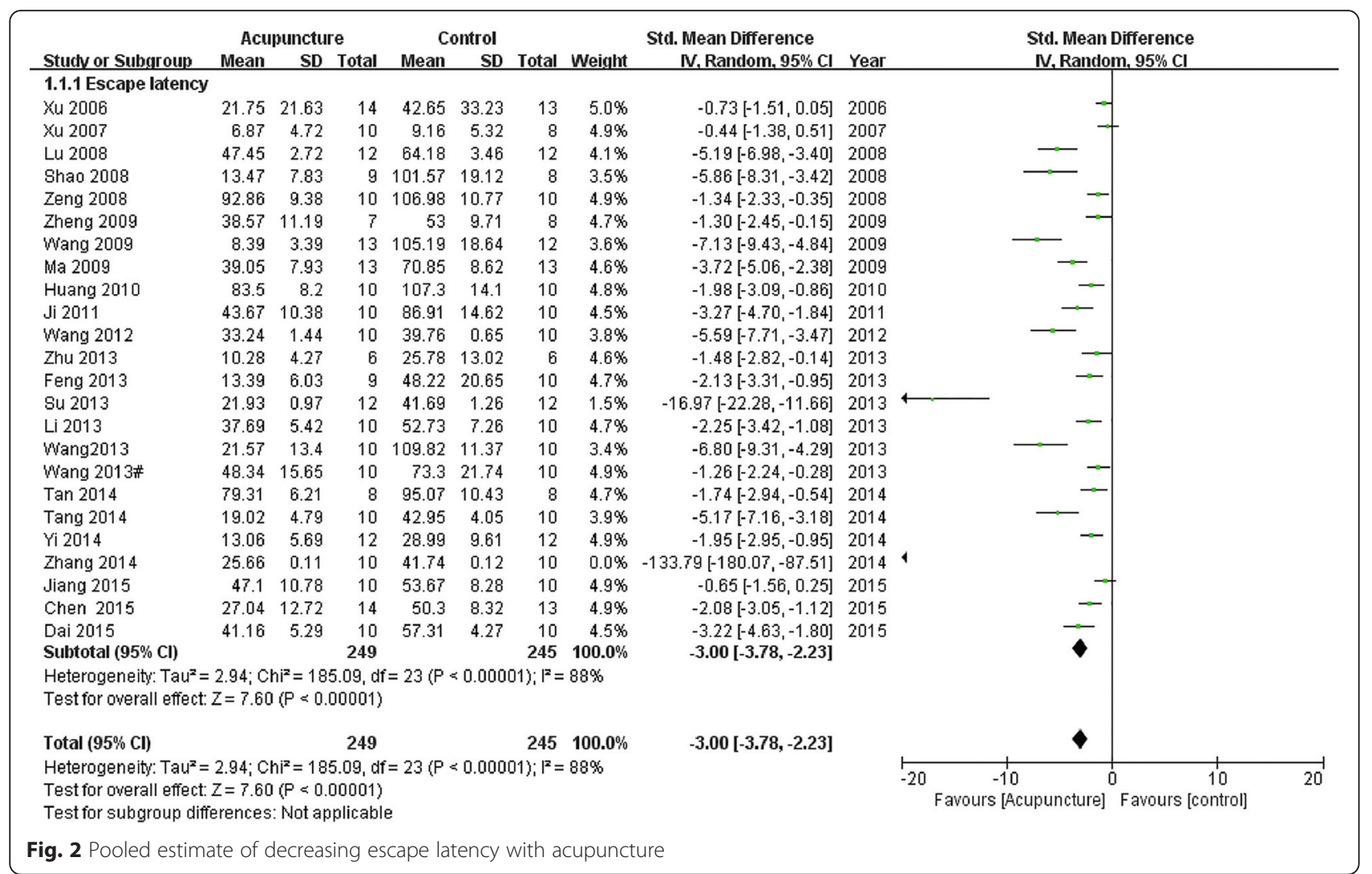

By analyzing different ways causing AD models, we found that $\mathrm{AD}$ models caused by $\mathrm{D}$-gal and $\mathrm{A} \beta$ were more sensitive to acupuncture than other ways (SMD -16.97, $95 \%$ CI: $-22.28 \sim-11.66$, Fig. 6e). By analyzing different ways causing VD models, we found that VD models caused by $2-\mathrm{VO}$ were more sensitive to acupuncture than other ways (SMD -3.12, $95 \%$ CI: $-5.96 \sim-0.28$, Fig. 6f). Different weight and age of rats were included in these studies. We found that rats whose weight ranges from 18 to $35 \mathrm{~g}$ (SMD -3.4, $95 \% \mathrm{CI}$ : $-5.05 \sim-1.75$, Fig. $6 \mathrm{~g}$ ) and rats whose age ranges from 2 to 4 months old (SMD $-2.86,95 \%$ CI: $-4.43 \sim-1.29$,

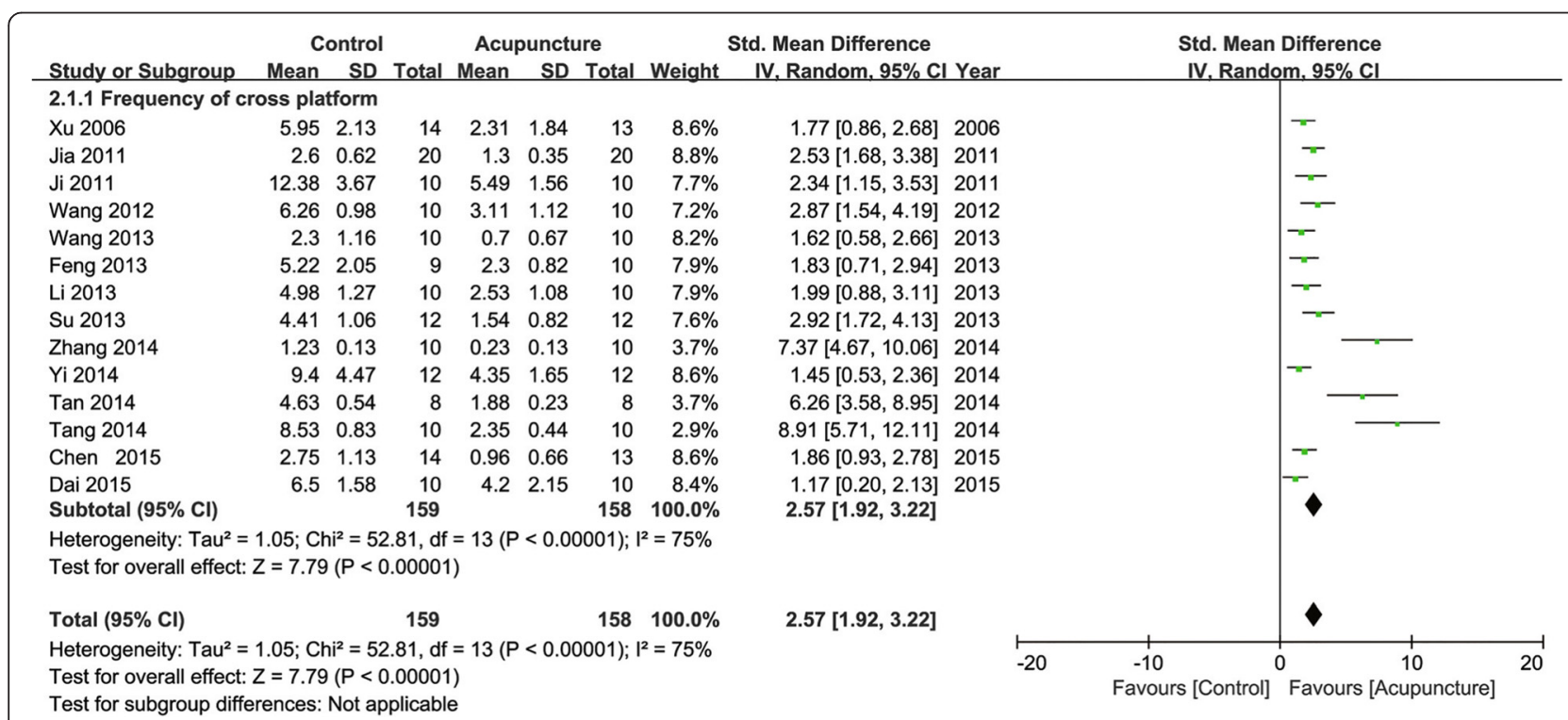

Fig. 3 Pooled estimate of increasing frequency of cross platform with acupuncture 


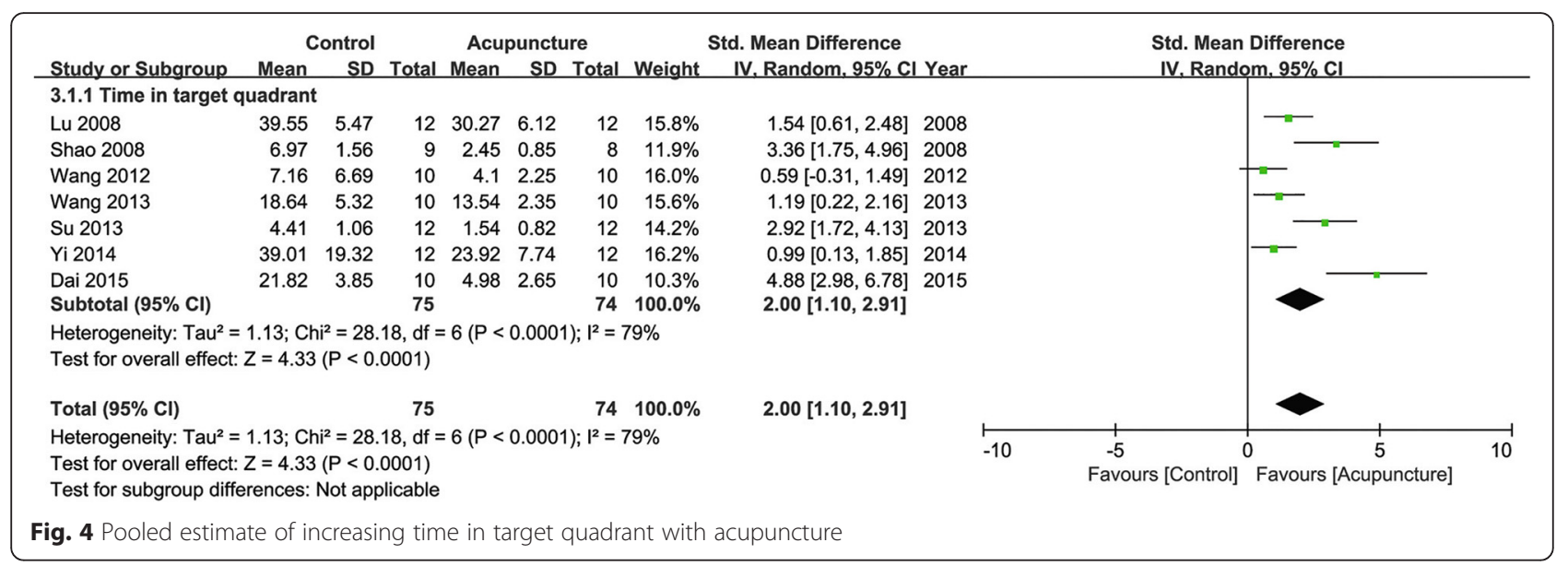

Fig. 6h) were more sensitive to acupuncture for improvement of frequency of escape latency.

In the subgroup analysis of frequency of cross platform, the electroacupuncture was more effective than manual acupuncture (SMD 2.72, 95 \% CI: 1.91 -3.52, Fig. 7a). Compared with unpublished articles, published articles showed more obvious changes of frequency of cross platform caused by acupuncture treatment (SMD 2.74, $95 \%$ CI: $1.96 \sim 3.53$, Fig. 7b). According to different varieties of experimental animals, Wister rats were more sensitive to acupuncture than SD rats and AKR rats (SMD3.31, $95 \%$ CI: 1.47 $\sim 5.16$, Fig. 7c). Compared with AD models, VD models got more improvement of frequency of cross platform (SMD 2.42, 95 \% CI: $1.60 \sim 3.24$, Fig. 7d). By analyzing different ways causing AD models, we found that SAMP8 models were more sensitive to acupuncture (SMD 3.04, 95 \% CI: $0.65 \sim 5.42$, Fig. 7e). By analyzing different ways causing VD models, we found that MCAO models were more sensitive (SMD 6.26, $95 \%$ CI: $3.58 \sim 8.95$, Fig. 7f). Different weight and age of rats were included in these studies. We found that rats whose weight ranges from 160 to 320 g (SMD 2.52, 95 \% CI: $1.86 \sim 3.17$, Fig. $7 g$ ) and rats whose age ranges from 2 to 4 months (SMD4.06, $95 \%$ CI: $2.30 \sim 5.83$, Fig. $7 \mathrm{~h}$ ) were more sensitive to acupuncture for improvement of frequency of cross platform.

In the subgroup analysis of time in target quadrant, the efficacy of manual acupuncture was better (SMD 2.94, $95 \%$ CI: $-0.67 \sim 6.55$, Fig. 8a). There were no unpublished articles using time in target quadrant as outcome indexes. According to different varieties of experimental animals, SD rats were more sensitive to acupuncture than Wister rats and AKR rats (SMD2.33, 95 \% CI: $0.77 \sim 3.89$, Fig. 8b). Compared with AD models, VD models got more improvement of time in target quadrant (SMD 3.36, 95 \% CI: 1.75 4.96, Fig. 8c). By analyzing different ways making models of $\mathrm{AD}$, we found that $\mathrm{AD}$ models caused by $\mathrm{D}$-gal and $\mathrm{A} \beta$ were more sensitive to acupuncture (SMD 2.92, 95 \% CI: $1.72 \sim 4.13$, Fig. 8d). 4-VO was the only way to be included causing VD models and using time in target quadrant as one of outcome indexes. Different weight and age of rats were included in these studies. We found that rats whose weight ranges from 18 to $35 \mathrm{~g}$ (SMD 3.10, $95 \% \mathrm{CI}:-0.16$ 6.37, Fig. 7e) and rats whose age ranges from 6 to 9 months old (SMD 2.31, 95 \% CI: $0.65 \sim 3.98$, Fig. 7f) were more sensitive to acupuncture for improvement of time in target quadrant.

These results were mostly inconsistent in three subgroup analyses. It may be caused by studies of low quality, publication bias or other reasons.

\section{Discussion}

To our knowledge, this is the first systematic review and meta-analysis to explore the efficacy of acupuncture for improving learning and memory in animal experiments with the results of Morris water maze test as the outcome measures. This systematic review and meta-analysis indicated that acupuncture could reduce time of escape latency, decrease frequency of cross platform and increase the time in target quadrant in animal model of learning and memory impairment. It suggests that acupuncture has a potential role in improving learning and memory impairment in animal models.

This review made a more detailed description of the acupuncture treatment procedure, including acupoint selection, stimulation methods, and treatment duration. The variation in the acupuncture protocol 
Table 3 Summary of proposed mechanisms

\begin{tabular}{|c|c|}
\hline Study & Findings or proposed mechanisms \\
\hline Bao 2014 [18] & $\begin{array}{l}\text { - Reduced apoptosis of hippocampal neurons } \\
\text { - Promoted restoration of damaged nerve cells }\end{array}$ \\
\hline Chen 2015 [20] & - Reduced Nogo-A and NgR \\
\hline Chen 2006 [21] & $\begin{array}{l}\text { - Increased GABA } \\
\text { - Reduced Glu }\end{array}$ \\
\hline Dai 2015 [22] & - Increased NEP \\
\hline Hou 2013 [23] & $\begin{array}{l}\text { - Increased MR } \\
\text { - Reduced GR }\end{array}$ \\
\hline Gao 2012 [24] & - Increased DA, 5-HT and NE \\
\hline Huang 2010 [25] & - Increased BDNF \\
\hline Ji 2011 [26] & $\begin{array}{l}\text { - Reduced MDA } \\
\text { - Increased SOD }\end{array}$ \\
\hline Jia 2011 [27] & $\begin{array}{l}\text { - Increased Syp, PKC, NMDAR and PKC mRNA } \\
\text { - Reduced mGluRs }\end{array}$ \\
\hline Wang 2012 [28] & $\begin{array}{l}\text { - Increased NOS } \\
\text { - Reduced MAO }\end{array}$ \\
\hline Lin 2008 [29] & - Increased NMDAR-2BmRNA \\
\hline Luo 2007 [30] & - Decreased NO \\
\hline Ma 2009 [31] & - Increased CTGF protein and mRNA \\
\hline Zhang 2014 [32] & - Increased InsR mRNA \\
\hline Niu 2009 [33] & - Increased SS and AVP \\
\hline Su 2013 [34] & $\begin{array}{l}\text { - Reduced MDA, P53 and P21 } \\
\text { - Increased SOD }\end{array}$ \\
\hline Tan 2014 [35] & - Increased GAP-43 and c-fos \\
\hline Tang 2014 [36] & - Increased CHAT protein \\
\hline Wang 2013 [37] & - Reduced GSK-3 $\beta$ \\
\hline Wang 2009 [38] & $\begin{array}{l}\text { - Reduced ET } \\
\text { - Increased CGRP }\end{array}$ \\
\hline Hong 2014 [39] & - Increased CX43, CX32 and CX36 \\
\hline Xu 2006 [40] & $\begin{array}{l}\text { - Increased } \mathrm{BCl}-2 \text { protein } \\
\text { - Reduced Bax protein and mRNA }\end{array}$ \\
\hline Xu 2007 [41] & - Reduced IL-1 $\beta$ and TNF- $a$ \\
\hline Yi 2014 [42] & $\begin{array}{l}\text { - Increased SOD, PKA and pCREB } \\
\text { - Reduced MDA and c-fos }\end{array}$ \\
\hline Feng 2013 [44] & - Increased GluR2 \\
\hline Li 2013 [45] & - Reduced MMP-2 and MMP-9 \\
\hline Zheng 2009 [47] & $\begin{array}{l}\text { - Increased ChAT } \\
\text { - Reduced TchE }\end{array}$ \\
\hline Li 2012 [48] & - Reduced neuron loss \\
\hline Li 2014 [49] & - Increased BDNF \\
\hline Lee 2014 [50] & $\begin{array}{l}\text { - Stimulated cholinergic enzyme activities } \\
\text { - Regulated BDNF and CREB expression }\end{array}$ \\
\hline Zhu 2013 [51] & - Up-regulated mTOR and elF4E \\
\hline Lu 2014 [52] & - Increased Fos expression \\
\hline Li 2012 [53] & $\begin{array}{l}\text { - increased Bcl-2mRNA } \\
\text { - decreased caspase-3 }\end{array}$ \\
\hline Guo 2015 [54] & - Down-regulated Notch1 and Hes1 mRNA \\
\hline Jiang 2015 [55] & - Increased the level of uptake rate of glucose \\
\hline Shao 2008 [56] & - Regulated the amount of AVP, SS, and $\beta$-EP \\
\hline
\end{tabular}

Table 3 Summary of proposed mechanisms (Continued)

\begin{tabular}{|c|c|}
\hline Liu 2013 [57] & $\begin{array}{l}\text { - Reduced MDA } \\
\text { - Increased SOD }\end{array}$ \\
\hline Li 2015 [58] & $\begin{array}{l}\text { - Increased the pyramidal neuron number } \\
\text { - Decreased the number of astrocytes }\end{array}$ \\
\hline Lu 2008 [59] & - Increased NCAM and ST8Siall/IVmRNA \\
\hline
\end{tabular}

Nogo-A neurite growth inhibitor-A, NgR neurite growth inhibitor receptor, GABA $\gamma$-aminobutyric acid, Glu glutamic acid, NEP neutral endopeptidase, $M R$ mineralocorticoid receptor, GR glucocorticoid receptor, DA dopamine, 5-HT 5-hydroxytryptamine, $N E$ norepinephrine, $B D N F$ brain-derived neurotrophic factor, MDA malondialdehyde, SOD superoxide dismutase, SYP synaptophysin, PKC Protein kinase C, NMDAR N-methyl-D-aspartate receptor, mGluRs metabolism glutanic acid acceptor, NOS nitric oxide synthase, MAO monoamine oxidase, NO nitric oxide, CTGF connective tissue growth factor, Ins $R$ insulin receptor, SS somatostatin, AVP arginine vasopressin, GAP-43 Growth Associated Protein-43, CHAT choline acetyl transferase, GSK-3 $\beta$ glycogen synthase kinase-3 $3, E T$ endothelia, CGRP calcitonin gene-related peptide, $C X$ connexin, IL interleukin, TNF tumor necrosis factor, $P C R E B$ phosphorylated CAMP-response element binding, MMP metal matrix proteinase, ChAT choline acetyltransferase, $T C h E$ total cholinesterase, CREB CAMP response element-binding protein, $m T O R$ mammalian target of rapamycin, elF4E eukaryotic translation initiation factor $4 \mathrm{E}$, NCAM neural cell adhesion molecule

might contribute to the heterogeneity in treatment outcome between studies. The most common acupoints in acupuncture treatment for improving learning and memory were GV20 (baihui), ST36 (zusanli), GV14 (dazhui), BL23 (shenshu), BL17 (geshu) and CV17 (danzhong). GV20 and GV14 are Governor Vessel acupoints. BL23 and BL17 are Bladder Meridian of Foot-Taiyang acupoints. This result suggests the possible importance of Governor Vessel and Bladder Meridian of Foot-Taiyang for learning and memory. About one-third of included studies used manual acupuncture, and twothird used electroacupuncture. We have found that continuous waves, $2-4 \mathrm{~Hz}$ of frequency and $1-2 \mathrm{~mA}$ of current density are the most commonly used stimulus parameters of electroacupuncture and twirling slowly is commonly used in manual acupuncture groups. Thus it can be seen that mild stimulation which means the reinforcing method in Traditional Chinese Medicine theory is adopted widely for improving learning and memory.

In the acupuncture study on animal model, setting sham acupuncture as control is very important and difficult. The importance of sham acupuncture is that it can help to clear the effectiveness of acupuncture after excluding placebo effects. While popular sham ways are mainly skin penetration. Except for placebo effects, it can also produce biological effects. Therefore, the true effect of acupuncture may be underestimated when compared to sham acupuncture [60].

Acupuncture has been known as an effective therapy for learning and memory impairment appearing in Alzheimer's disease, vascular dementia, and so on 


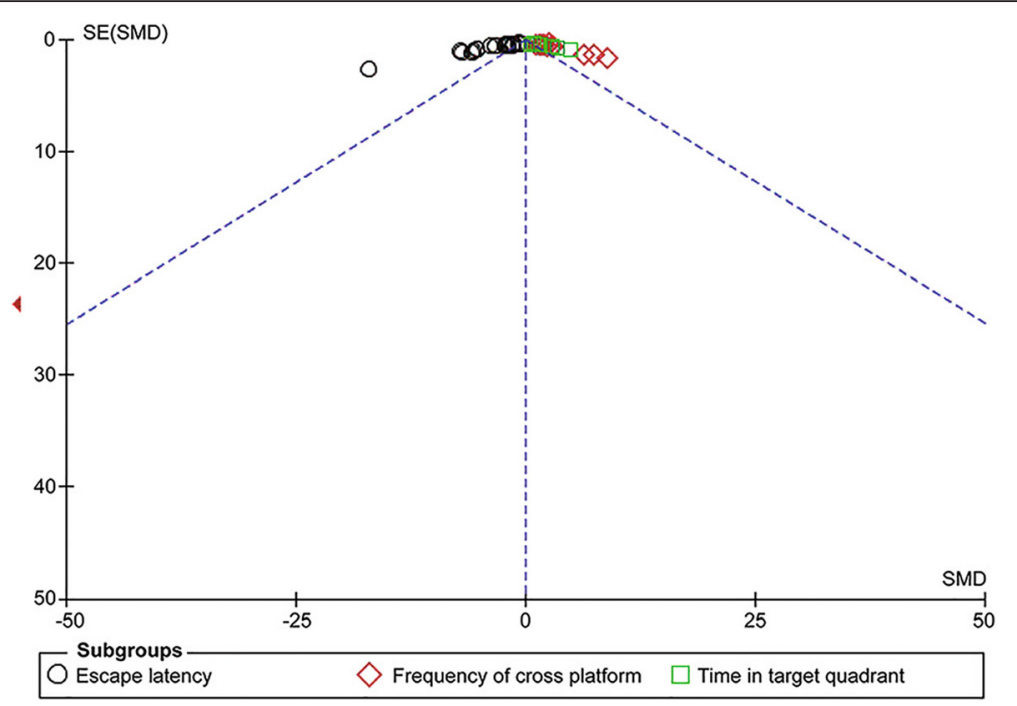

Fig. 5 Funnel plot for effectiveness of acupuncture on escape latency, frequency of cross platform and time in target quadrant

[5-8]. The mechanism of acupuncture on improving learning and memory ability remains unclear. It is recognized that learning and memory are associated with cerebral cortex and hippocampus closely [40]. Modern medical research suggests that acupuncture may reduce nerve apoptosis and necrosis to protect cortex and hippocampus neurons through different aspects, which include decreasing the level of cytokines in hippocampus [41], adjusting the neurotrophic factors and cholinergic system [42, 47], inhibiting the expression of matrix metalloproteinase(MMP) [45], and so on. Additionally, it has been suggested that acupuncture can increase the activity of superoxide dismutase (SOD) and decrease the level of malondialdehyde (MDA) in brain to improve the antioxidant capacity and reduce brain tissue damage caused by free radical [58].

There are some limitations to this review. Firstly, our search only included Chinese and English articles and excluded those articles published in other languages. At the same time, we only included manual acupuncture and electroacupuncture and excluded some special acupuncture therapies, such as scalp acupuncture, auricular acupuncture, abdominal acupuncture, and so on. It may cause selective bias. Secondly, the total number of studies and the total sample size were too small for reliable. We have performed comprehensive literature search (six databases) and conducted extensive searches through other sources. But no more studies were found. Thirdly, articles which reported negative results may not be popular to publish so that the effectiveness of published articles would be better than those unpublished. Therefore, the effectiveness of acupuncture for improving learning and memory may be overstated. Fourthly, the quality of included studies was very low, so that it had significant impacts on the outcomes of the meta-analysis.

Based on the above limitations, more other language articles and special acupuncture treatment means should be included in the future systematic. Meanwhile, control of temperature, random allocation to treatment or control, blinded building of model, assessment successful model building and blinded assessment of outcome should be pay attention to in the future animal or clinical studies. Not only positive results, but also negative should be reported in the future animal or clinical studies.

In addition, some implications are also brought out after analyzing affecting factors of outcome indexes. Manual acupuncture showed more effective to escape latency and time in target quadrant than electroacupuncture. And they had roughly the same impact on frequency of cross platform. But in the present study, only 13 out of 42 studies performed manual acupuncture and the rest performed electroacupuncture. Because electroacupuncture is easier to control, standard and objectively measure than manual acupuncture [12], so it is used widely in clinical and experimental researches. Therefore, it remains unknown which means of acupuncture is more effective and convenient for people's learning and memory impairment. Other impact factors do not show any universal regularity through histogram analysis. It need be solved by higher quality studies, lower publication bias, and so on. 

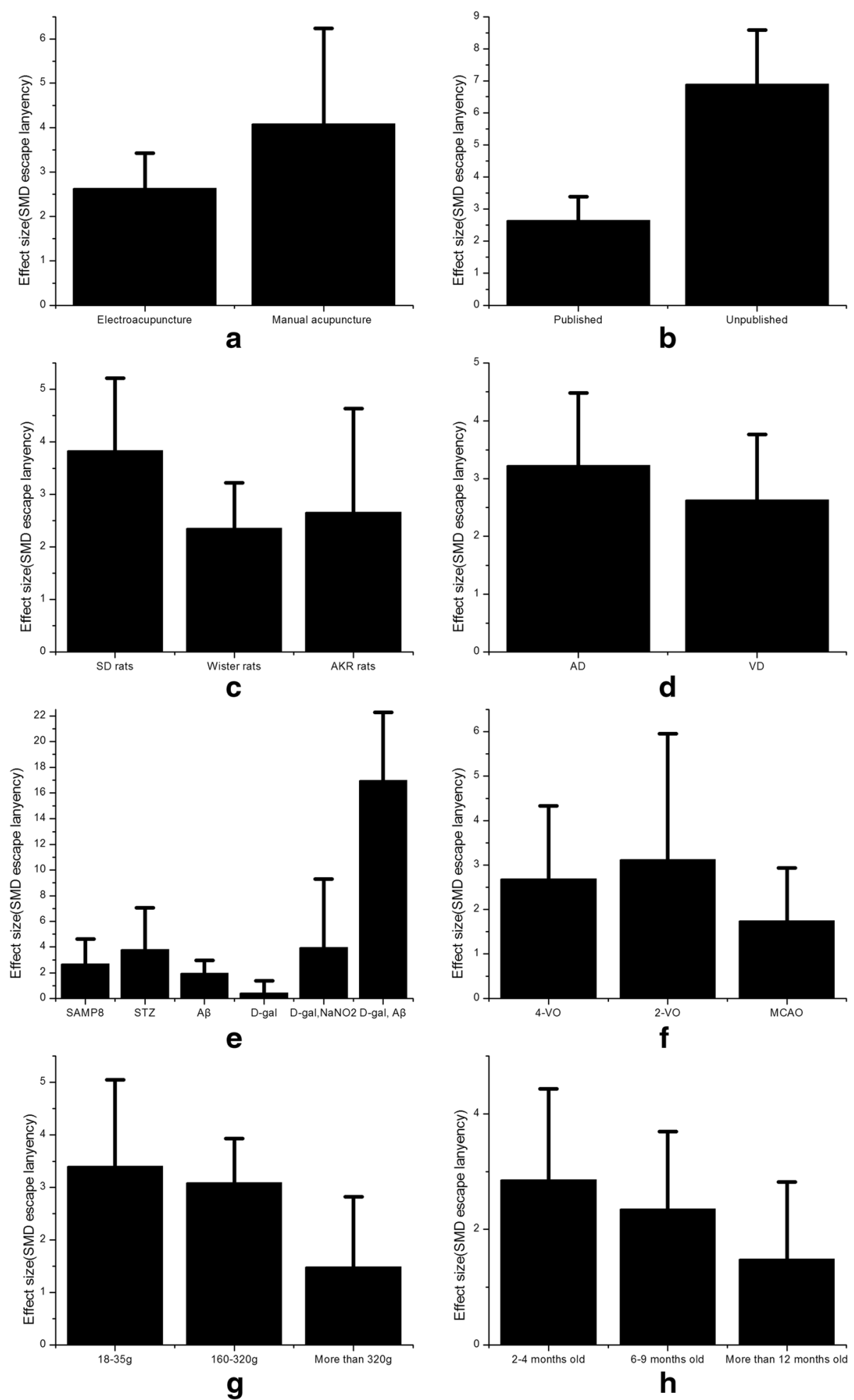

Fig. $\mathbf{6}$ Subgroup analysis according to escape latency. a The effect of manual acupuncture and electroacupuncture on the estimate of improvement in escape latency. $\mathbf{b}$ The impact of published articles compared with unpublished articles on the estimate of improvement in escape latency. $\mathbf{c}$ The type of strain on the estimate of improvement in escape latency. $\mathbf{d}$ The sensitiveness of AD model compared with VD model on the estimate of improvement in escape latency. $\mathbf{e}$ The different ways making AD model on the estimate of improvement in escape latency. $\mathbf{f}$ The different ways making VD model on the estimate of improvement in escape latency. $\mathbf{g}$ The different weights on the estimate of improvement in escape latency. $\mathbf{h}$ The different age on the estimate of improvement in escape latency 

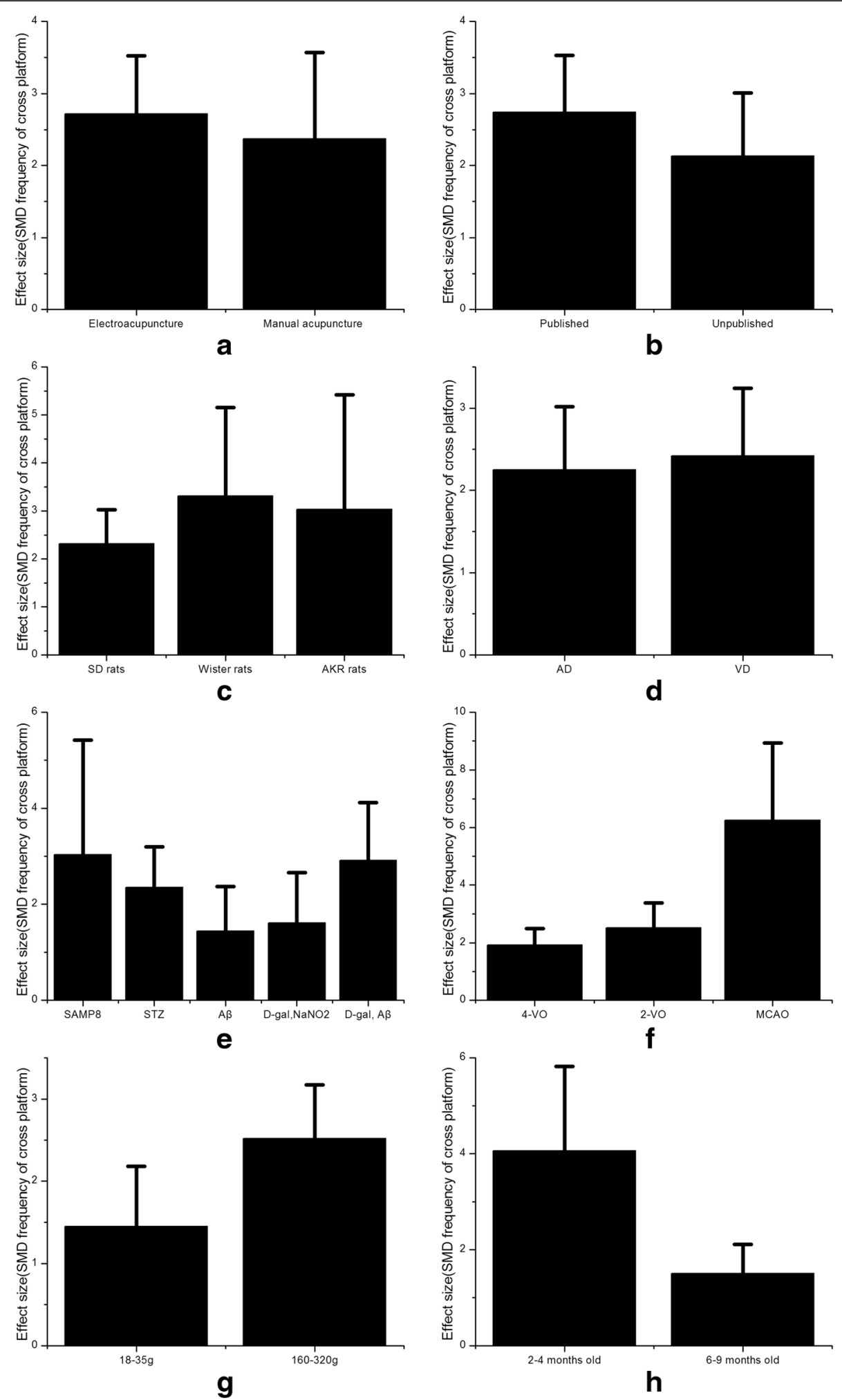

Fig. 7 (See legend on next page.) 
(See figure on previous page.)

Fig. 7 Subgroup analysis according to frequency of cross platform. a The effect of manual acupuncture and electroacupuncture on the estimate of improvement in frequency of cross platform. $\mathbf{b}$ The impact of published articles compared with unpublished articles on the estimate of improvement in frequency of cross platform. $\mathbf{c}$ The type of strain on the estimate of improvement in frequency of cross platform. $\mathbf{d}$ The sensitiveness of AD model compared with VD model on the estimate of improvement in frequency of cross platform. e The different ways making AD model on the estimate of improvement in frequency of cross platform. $\mathbf{f}$ The different ways making VD model on the estimate of improvement in frequency of cross platform. $\mathbf{g}$ The different weights on the estimate of improvement in frequency of cross platform. $\mathbf{h}$ The different age on the estimate of improvement in frequency of cross platform

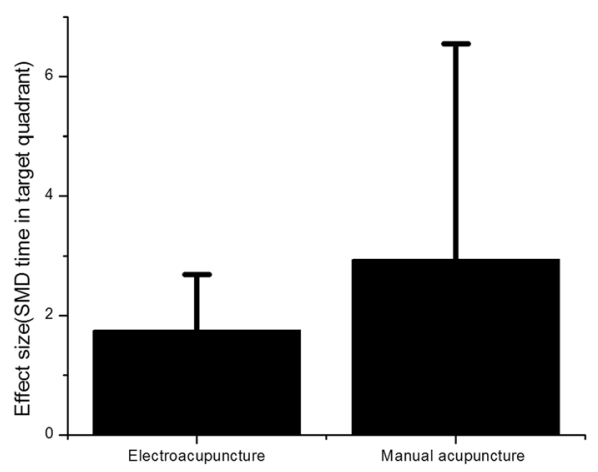

a
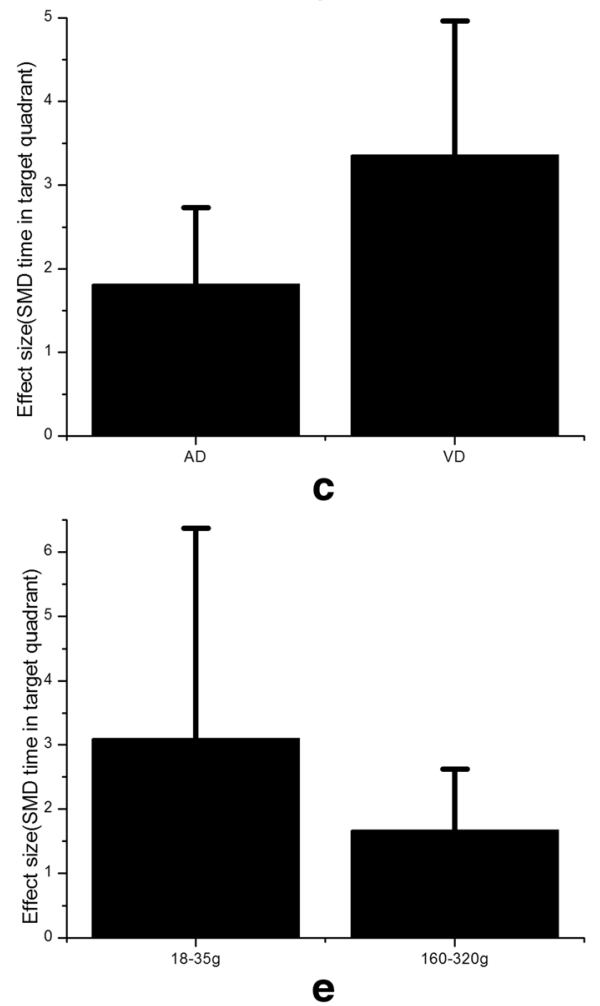
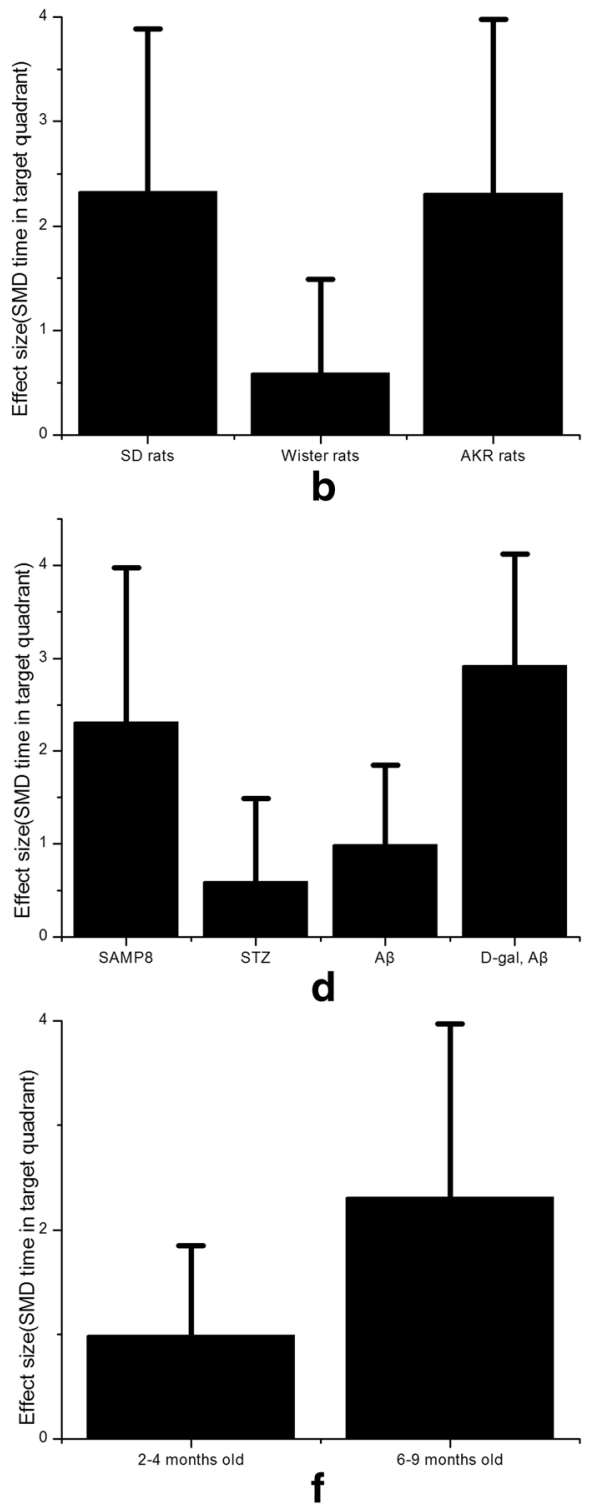

Fig. 8 Subgroup analysis according to time in target quadrant. a The effect of manual acupuncture and electroacupuncture on the estimate of improvement in time in target quadrant. $\mathbf{b}$ The type of strain on the estimate of improvement in time in target quadrant. $\mathbf{c}$ The sensitiveness of AD model compared with VD model on the estimate of improvement in time in target quadrant. $\mathbf{d}$ The different ways making AD model on the estimate of improvement in time in target quadrant. e The different weights on the estimate of improvement in time in target quadrant. $\mathbf{f}$ The different age on the estimate of improvement in time in target quadrant 


\section{Conclusion}

In animal model, acupuncture has a potential role in improving learning and memory ability. But it is still ambiguous that which stimulating mode (manual acupuncture or electroacupuncture) is more effective. Low quality of studies and larvaceous publication bias may reduce persuasiveness of positive results and should be solved in the future.

\section{Acknowledgements}

We would like to express our gratitude and thanks to Antoine Grellet (Nanjing University of Chinese Medicine) and Xin Cao (Department of Pharmacology, Faculty of Medicine, Toho University) for editing of English.

\section{Funding}

This work was supported by grants from the National Natural Science Foundation of China (No.81202743, 81574062, 81303019, 81403478). The funders had no role in study design, data collection and analysis, decision to publish, or preparation of the manuscript.

\section{Availability of data and materials}

The datasets supporting the conclusions of this article are included within the article.

\section{Authors' contributions}

Conceived and designed the experiments: KYH SL SFL. Performed the experiments: KYH SL. Analyzed the data: MLY SFL SPF. Contributed reagents/ materials/analysis tools: MLY SFL. Wrote the paper: KYH SL XC. Revised the manuscript: SFL SPF XC. Agreed with the manuscript's results and conclusions: KYH SL MLY SPF XC SFL. All authors read and approved the final manuscript.

\section{Competing interests}

The authors declare that they have no competing interests.

\section{Consent for publication}

Not applicable.

\section{Ethics approval and consent to participate}

Not applicable.

Received: 2 March 2016 Accepted: 17 August 2016

Published online: 19 August 2016

\section{References}

1. Brem AK, Ran K, Pascual-Leone A. Learning and memory. Handb Clin Neurol. 2013;116:693-737.

2. Colciago A, Casati L, Negri-Cesi P, Celotti F. Learning and memory: Steroids and epigenetics. J Steroid Biochem Mol Biol. 2015;150:64-85.

3. Baudry $M, B i X$. Learning and memory: an emergent property of cell motility. Neurobiol Learn Mem. 2013;104:64-72. doi:10.1016/j.nlm.2013.04. 012.

4. Gallistel CR, Balsam PD. Time to rethink the neural mechanisms of learning and memory. Neurobiol Learn Mem. 2014;108:136-44.

5. Sindi S, Mangialasche F, Kivipelto M. Advances in the prevention of Alzheimer's Disease. F1000Prime Rep. 2015:7:50.

6. Wang B, Zhong Y, Yan H, Cui L. Meta-analysis of plasma homocysteine content and cognitive function in elderly patients with Alzheimer's disease and vascular dementia. Int J Clin Exp Med. 2014;7:5118-23.

7. Kim C, Sohn JH, Jang MU, Kim SH, Choi MG, Ryu OH, et al. Association between visit-to-visit glucose variability and cognitive function in aged type 2 diabetic patients: a cross-sectional study. PLoS One. 2015;10, e0132118.

8. Duerden EG, Taylor MJ, Lee M, McGrath PA, Davis KD, Roberts SW. Decreased sensitivity to thermal stimuli in adolescents with autism spectrum disorder: relation to symptomatology and cognitive ability. J Pain. 2015;16:463-71.

9. Ritter A, Pillai JA. Treatment of vascular cognitive impairment. Curr Treat Options Neurol. 2015;17:367. doi:10.1007/s11940-015-0367-0.
10. Versijpt J. Effectiveness and cost-effectiveness of the pharmacological treatment of Alzheimer's disease and vascular dementia. J Alzheimers Dis. 2014;42 Suppl 3:S19-25.

11. Wang LP, Zhang XY, Liu N, Ma ZZ, Fang DS. Comparison of integrated traditional Chinese and western medicine therapy on vascular cognitive impairment with no dementia. Genet Mol Res. 2015;14:4896-902.

12. Wang WW, Xie CL, Lu L, Zheng GQ. A systematic review and meta-analysis of Baihui (GV20)-based scalp acupuncture in experimental ischemic stroke. Sci Rep. 2014;4:3981.

13. Zhou JW, Zhang AR, Qiu L, Huang S, Wang WC, Hu YH, et al. Cognitive impairment in earthquake brain injury treated with comprehensive program of acupuncture and rehabilitation: a randomized controlled trial. Zhongguo Zhen Jiu. 2014:34:105-9. article in Chinese.

14. Zhang H, Zhao L, Yang S, Chen Z, Li Y, Yang Y, et al. Clinical observation on effect of scalp electroacupuncture for mild cognitive impairment. J Tradit Chin Med. 2013;33:46-50.

15. Feng $Y$, Bai L, Ren Y, Chen S, Wang H, Zhang W, et al. FMRI connectivity analysis of acupuncture effects on the whole brain network in mild cognitive impairment patients. Magn Reson Imaging. 2012;30:672-82.

16. Chou P, Chu H, Lin JG. Effects of electroacupuncture treatment on impaired cognition and quality of life in Taiwanese stroke patients. J Altern Complement Med. 2009;15:1067-73.

17. Murphy SP, Murphy AN. Pre-clinical systematic review. J Neurochem. 2012; 115:805

18. Bao WY, Jiao S, Lu J, Tu Y, Song YZ, Wu Q, et al. Effect of electroacupuncture intervention on learning-memory ability and injured hippocampal neurons in depression rats. Zhen Ci Yan Jiu. 2014;39:136-41. article in Chinese.

19. Zeng XF, Fan TC, Wang MK, Xue SF, Zhang XL, Zhong BL, et al. Influence of electric - needling acupoint Siguan on learning ability and memory of model rats with Alzheimer's disease. Medical Journal of the Chinese People' s Armed Police Forces. 2008;19:781-4. article in Chinese.

20. Chen JX, Lin YK, Wu YN, Zhou YP, Lin RH, Tao J, et al. Effects of electroacupuncture on learning and memory and the expression of Nogo-A and $\mathrm{NgR}$ in rats with focal cerebral ischemia-reperfusion. Chinese Journal of Rehabilitation Medine. 2015;30:219-23. article in Chinese.

21. Chen SL. The effect of acupuncture on learning and memory impairment in hypoxiic-ischemic brain damage [Ph.D]. China Academy of Chinese Medical Sciences. 2006. article in Chinese.

22. Dai SS, Dong KL, Zhu H. Effects of "bushenen huoxue" acupuncture on the ability learning and memory and the expression of NEP in the hippocampal CA1 region of rapid aging SAMP8 mouse models. Journal of Hunan University of Chinese Medicine. 2015;35:60-3. article in Chinese

23. Hou LQ, Liu S, Xiong KR. Effects of electroacupuncture intervention on behavior changes and hippocampal glucocorticoid receptor and mineralocorticoid receptor expression levels in post traumatic stress disorder rats. Zhen Ci Yan Jiu. 2013;38:140-5. article in Chinese.

24. Gao HY. The research on the spatial memory influence of electroacupuncture on the aged AD rats [M.Sc]. China: Chengdu University of Traditional Chinese Medicine; 2012. article in Chinese.

25. Huang PP, Ma J, Wang YC, Gan SY, Li H, Lei T. Experiment research for the effect of Taizhong and Fengfu on PD rats' learning-memory and BDNF. Traditional Chinese Medicine Journal. 2010;9:55-7. article in Chinese.

26. Ji JF, Guan JJ, Wu SW. Effect of acupuncture on superoxide dismutase and malondialdehyde in rat with vascular dementia rat. Hebei Journal of Traditional Chinese Medicine. 2011;33:1554-6. article in Chinese.

27. Jia XM. Effect of electro-acupuncture therapy on VD mouse' learningmemory and seahorse synapse plasticity [M.Sc]. China: Ningbo University; 2011. article in Chinese.

28. Wang JX, Zhang HN, Zhang YY, Han TZ, Liu Y, Xu Z, et al. The effect of Baihui, Dazhui and Zusanli on AD rats' learning-memory, MAO and NOS. Information on Traditional Chinese Medicine. 2012;29:94-6. article in Chinese.

29. Lin HJ, Wang W. Effect of electroacupuncture on NMDAR-2 BmRNA expression in hippocampal tissue in vascular dementia rats. Zhen Ci Yan Jiu. 2008:33:301-5. article in Chinese.

30. Luo R, Yan B, He LL. he influences of electro -acupuncture on learn and memory abilities and nitric oxide in cerebrum of vascular dementia rats. Chinese Journal of Rehabilitation Medicine. 2007;22:500-2. article in Chinese.

31. Ma P, Ji Z, Yu H, Chu YR. Effect of electroacupuncture on learning and memory in diabetic rats and the expression of connective tissue growth 
factor in hippocampus. Journal of Xi an Jiaotong University(Medical Sciences). 2009;30:30-2. article in Chinese.

32. Zhang QA, Sun XH, Gao H, Lin JJ, Fang L, Li BW, et al. The effect of acupuncture on learning and memory ability of SAMP8 mice and the expression of insulin receptor (InsR) mRNA in hippocampus. Journal of Gansu College of Traditional Chinese Medicine. 2014;31:1-4. article in Chinese.

33. Niu WM, Liu ZB, Yang XH, Niu XM, Wang Y. Effect of "Xiusanzhen" on learning-memory ability and hppocampal somatostatin and arginine vesopressin contents in vascular dementia rats. Zhen Ci Yan Jiu. 2009;34: 106-9. article in Chinese.

34. Su Q, Xu D. Electro-acupuncture treatment impact as well as the mechanisms of learning and memory abilities of $\mathrm{AD}$ rats. Chongqing Medical Journal. 2013;42:2444-8. article in Chinese.

35. Tan B, Duan XD, Yu Q. Effect of Electro-acupuncture on GAP-43 and c-fos of hippocampal neurons in ischemia side and relationship between effects and learning and memory in cerebral infarction rats. Journal of Chengdu University of Traditional Chinese Medicine. 2014;37:33-7. article in Chinese.

36. Tang $X$, Tang $C L$, Liu RJ. The effect of electroacupuncture on OVX rats' learning-memory and CHAT. Chin J Gerontol. 2014;34:1856-8. article in Chinese.

37. Wang DX. The effect of acupuncture on GSK-3 $\beta$, ACHE and antioxygenation [M.Sc]. China: Guiyang College of Traditional Chinese Medicine; 2013. article in Chinese.

38. Wang LF. The effect of acupuncture on vascular dementia rats bloodplasma content of CGRP, ET and neural cell of synaptic plasticity in the hippocampus. [M.Sc]. China: Shaanxi College of Traditional Chinese Medicine; 2009. article in Chinese.

39. Hong YZ, Zhang XJ, Hong L, Huang QR, Wu Q. Influence of acupuncture of "Changqiang" (GV 1)on learning-memory ability and gap junction-related protein expression in the prefrontal cortex in autism rats. Zhen Ci Yan Jiu. 2014;39:173-9. article in Chinese.

40. Xu GF, Li M. Effect of electroacapunchire on HO-1, BCl-2 and bax proteii expcession rats with vascular dementia. Liaoning Journal of Traditional Chinese Medicine. 2006;33:1510-512. article in Chinese.

41. Xu Y, Zhang ZX, Shen R, Wang XY, Li Y. Effect of electroacupuncture on ethology and cytokines of hippocampus in rats with dysmnesy. Zhen Ci Yan Jiu. 2007:32:88-92. article in Chinese.

42. Yi XF, Peng $L$, Zhang $Z Y$, Xiao $M, M u J P$, Xie $L Y$, et al. Influence of electroacupuncture on CAMP/PKA/CREB signal transduction pathways in Ap25-35 induced Alzheimer's disease model rats. Journal of Chinese Practical Diagnosis and Therapy. 2014;28:128-30. article in Chinese.

43. Yu Q, Hu L, Liu L, Wang J, Wang CH. Effect of electroacupuncturing Shenmen(HT7) on P300 of event-related potentials in rats of Wilson's disease. Journal of Sichuan of Traditional Chinese Medicine. 2014;32:62-5. article in Chinese.

44. Feng M, Xiong YY, Lu J, Zhang JS, Yu ML, Wu QF, et al. The effect of acupuncture on AMPA receptor expression in the hippocampus of senescence-accelerated mice is correlated with synaptic plasticity. Chinese Journal of Physical Medicine and Rehabilitation. 2013;35:677-81. article in Chinese.

45. Li ZF, Liu ZJ, Liu DY, Zhang HY. Effect of electric acupuncture on learning and memory and MMP-2,MMP-9 of hippocampal matrix in rats with Alzheimer's disease. China Modern Medicine. 2013;20:15-9. article in Chinese.

46. Wang LY, Nie K, Liu JF, Luo BH, Zhang XZ. Effect of acupuncture on signs of senescence and cognitive function in SAMP8 mice. Tianjin Journal of Traditional Chinese Medicine. 2013;30:349-52. article in Chinese

47. Zheng J, Zhou XM, Guo XX, Jin HZ. Effect of needling "Baihui" and "Taixi" on cognitive function of rat model with vascular dementia. Chinese Archives of Traditional Chinese Medicine. 2009;27:1037-9. article in Chinese.

48. Li G, Zhang X, Cheng H, Shang X, Xie H, Zhang X, et al. Acupuncture improves cognitive deficits and increases neuron density of the hippocampus in middle-aged SAMP8 mice. Acupunct Med. 2012;30:339-45.

49. Li X, Guo F, Zhang Q, Huo T, Liu L, Wei H, et al. Electroacupuncture decreases cognitive impairment and promotes neurogenesis in the APP/PS1 transgenic mice. BMC Complement Altern Med. 2014;14:37.

50. Lee B, Sur B, Shim J, Hahm DH, Lee H. Acupuncture stimulation improves scopolamine-induced cognitive impairment via activation of cholinergic system and regulation of BDNF and CREB expressions in rats. BMC Complement Altern Med. 2014;14:338.
51. Zhu Y, Zeng $Y$, Wang $X$, Ye X. Effect of electroacupuncture on the expression of mTOR and elF4E in hippocampus of rats with vascular dementia. Neurol Sci. 2013;34:1093-7.

52. Lu B, Ma Z, Cheng F, Zhao Y, Zhang X, Mao H, et al. Effects of electroacupuncture on ethanol-induced impairments of spatial learning and memory and Fos expression in the hippocampus in rats. Neurosci Lett. 2014;576:62-7.

53. Li M, Peng J, Song Y, Liang H, Mei Y, Fang Y. Electro-acupuncture combined with transcranial magnetic stimulation improves learning and memory function of rats with cerebral infarction by inhibiting neuron cell apoptosis. J Huazhong Univ Sci Technolog Med Sci. 2012;32:746-9.

54. Guo HD, Tian JX, Zhu J, Li L, Sun K, Shao SJ, et al. Electroacupuncture suppressed neuronal apoptosis and improved cognitive impairment in the $A D$ model rats possibly via downregulation of notch signaling pathway. Evid Based Complement Alternat Med. 2015:2015:393569.

55. Jiang J, Gao K, Zhou Y, Xu A, Shi S, Liu G, et al. Electroacupuncture treatment improves learning-memory ability and brain glucose metabolism in a mouse model of Alzheimer's disease: using morris water maze and micro-PET. Evid Based Complement Alternat Med. 2015;2015:142129.

56. Shao Y, Fu YQ, Qiu LH, Yan B, Lai XS, Tang CZ. Electropuncture influences on learning, memory, and neuropeptide expression in a rat model of vascular dementia. Neural Regen Res. 2008:3:267-71.

57. Li F, Yan CQ, Lin LT, Li H, Zeng XH, Liu Y, et al. Acupuncture attenuates cognitive deficits and increases pyramidal neuron number in hippocampal CA1 area of vascular dementia rats. BMC Complement Altern Med. 2015;15: 133.

58. Liu CZ, Lei B. Effect of acupuncture intervention on learning-memory ability and cerebral superoxide dismutase activity and malonaldehyde concentration in chronic fatigue syndrome rats. Zhen Ci Yan Jiu. 2013; 38:478-81. article in Chinese.

59. Lu SF, Shao X, Tang Y, Yin HY, Chen J, Yu SG. Effects of electroacupuncture on neural cell adhesion of synaptic plasticity in the hippocampus of SAMP8. Chinese Journal of Rehabilitation Medicine. 2008:23:1057-60. article in Chinese.

60. Shergis JL, Ni X, Jackson ML, Zhang AL, Guo X, Li Y, et al. A systematic review of acupuncture for sleep quality in people with insomnia. Complement Ther Med. 2016;26:11-20.

\section{Submit your next manuscript to BioMed Central and we will help you at every step:}

- We accept pre-submission inquiries

- Our selector tool helps you to find the most relevant journal

- We provide round the clock customer support

- Convenient online submission

- Thorough peer review

- Inclusion in PubMed and all major indexing services

- Maximum visibility for your research

Submit your manuscript at www.biomedcentral.com/submit 